\title{
Off-shell renormalization in the presence of dimension 6 derivative operators. Part III. Operator mixing and $\beta$ functions
}

\author{
D. Binosi ${ }^{a}$ and A. Quadri ${ }^{b}$ \\ ${ }^{a}$ European Centre for Theoretical Studies in Nuclear Physics and Related Areas (ECT*) \\ and Fondazione Bruno Kessler, \\ Villa Tambosi, Strada delle Tabarelle 286, I-38123 Villazzano TN, Italy \\ ${ }^{b}$ INFN - Sezione di Milano, \\ via Celoria 16, I-20133 Milano, Italy \\ E-mail: binosi@ectstar.eu, andrea.quadri@mi.infn.it
}

ABSTRACT: We evaluate the one-loop $\beta$ functions of all dimension 6 parity-preserving operators in the Abelian Higgs-Kibble model. No on-shell restrictions are imposed; and the (generalized) non-polynomial field redefinitions arising at one-loop order are fully taken into account. The operator mixing matrix is also computed, and its cancellation patterns explained as a consequence of the functional identities of the theory and powercounting conditions.

KEYwords: Beyond Standard Model, BRST Quantization, Effective Field Theories ArXIV EPRINT: 1904.06693 


\section{Contents}

1 Introduction 1

2 Notations and conventions 3

3 One-loop UV divergences 5

$\begin{array}{lll}4 & \text { Dimension six operators coefficients } & 7\end{array}$

5 Generalized field redefinitions $\quad 7$

$\begin{array}{ll}5.1 \text { GFRs in the target theory } & 11\end{array}$

6 Renormalization of gauge invariant operators 11

6.1 Pure external sources invariants 11

$\begin{array}{lll}6.2 & \text { Mixed field-external sources invariants } & 12\end{array}$

$\begin{array}{ll}\text { 6.3 Gauge invariants depending only on the fields } & 14\end{array}$

$\begin{array}{lll}7 & \text { Mapping to the target theory } & 15\end{array}$

8 One-loop mixing matrices 16

$\begin{array}{lll}9 & \text { Conclusions } & 19\end{array}$

A Functional identities in the $X$-theory $\quad 19$

$\begin{array}{lll}\text { A.1 ST identities } & 19\end{array}$

A.2 $X_{1,2}$-equations 20

$\begin{array}{lll}\text { A.3 Solving the } X_{1,2} \text {-equations } & 20\end{array}$

$\begin{array}{ll}\text { B The } b \text { - and the gauge ghost equation } & 20\end{array}$

$\begin{array}{ll}\text { C Propagators } & 21\end{array}$

C.1 The $X-\sigma$ sector $\quad 21$

$\begin{array}{ll}\text { C.2 The gauge and ghost sector } & 22\end{array}$

D List of gauge-invariant operators $\quad 22$

D.1 Pure external sources invariants 22

D.2 Mixed field-external sources invariants 23

D.3 Gauge invariants depending only on the fields 23

E On-shell reduction of dim. 6 field-dependent gauge invariant operators 24 


\section{Introduction}

New physics beyond the Standard Model (SM) can be characterized in a model independent and systematic fashion within the Effective Field Theories (EFTs) framework, in which the (renormalizable) tree-level SM action is supplemented with the terms $(k \geq 5)$

$$
S_{0}^{[k]}=\int \mathrm{d}^{4} x \sum_{i} c_{i}^{[k]} \mathcal{O}_{i}^{[k]}
$$

where $\mathcal{O}_{i}^{[k]}$ are $k$-dimensional operators whose dimension dictates the suppression of the corresponding coefficients $c_{i}^{[k]}$ in terms of powers of a high-energy scale $\Lambda$. The resulting Standard Model Effective Field Theory (SMEFT) action

$$
S_{0} \equiv \underbrace{\int \mathrm{d}^{4} x \mathscr{L}_{\mathrm{SM}}}_{\sum_{k=2}^{4} S_{0}^{[k]}}+\sum_{k \geq 5} S_{0}^{[k]},
$$

is not renormalizable in the usual (power counting) sense; it is, nevertheless, renormalizable in the modern sense [1], as all the divergences can be cancelled through the renormalization of the (infinite) number of terms in the bare action while respecting the symmetries of the theory.

When addressing operator mixing in such theories on-shell calculations are sufficient. Indeed while it has been known since a long time that there is ultraviolet (UV) mixing between gauge invariant and gauge variant (unphysical) operators (also known as 'alien' operators [2]), it has also been shown that such mixing can be made to vanish by a suitable choice of the basis in the space of local operators [3-6]; additionally, alien operators have been shown to be cohomologically trivial and therefore have vanishing on-shell correlators [3] (for a review see also [7]). This fact is at the basis of recent computations in the literature [8-11] as it implies that for certain purposes, e.g., when evaluating anomalous dimensions and/or $S$-matrix elements, one can consider only on-shell inequivalent operators [12].

A separate issue, however, is the evaluation of the $\beta$-functions of the theory. For this purpose one needs to extend the approach adopted in the power-counting renormalizable case [13-15] to EFTs; in particular, one must work out a procedure to fix the generalized field redefinitions (GFRs) that do arise in these models. Here 'generalized' means that, at variance with the power-counting renormalizable case, these redefinitions are not linear in the quantum fields (in fact, not even polynomial already at one-loop order, as we will show). The matching of the couplings order by order in the loop expansion, once the GFRs' effects are taken into account, is the next technical step required to match the model with its UV completions while respecting the locality of the low energy theory also at higher loop orders, since it allows to unequivocally fix the correct counter-terms needed to subtract overlapping divergences with local counter-terms.

To attain these goals, in [16] it has been developed a general theory for the recursive subtraction of off-shell UV divergences order by order in the loop expansion applicable to 
EFTs displaying a spontaneously broken symmetry phase. This is achieved by solving the Slavnov-Taylor (ST) identity to all-orders, which allows in turn to disentangle the gaugeinvariant contributions to the off-shell one-particle irreducible (1PI) amplitudes from those associated with the gauge fixing and field redefinitions, which, in a general EFT, can be (and indeed are) non polynomial (and cannot obviously be accessed staying on-shell). Next, in [17] this algebraic technique has been applied to study the Abelian Higgs-Kibble (HK) model in the presence of the dimension 6 operator $(g / \Lambda) \phi^{\dagger} \phi\left(D_{\mu} \phi\right)^{\dagger} D^{\mu} \phi$, which, giving rise to an infinite number of one-loop divergent diagrams, maximally violates power counting. In particular, the complete renormalization of all the radiatively generated dimension 6 operators has been carried out together with the determination of the full $g$-dependence of the $\beta$-function coefficients.

Before moving on to consider the full dimension 6 SMEFT [18], there is just one aspect that has been left out in the study of its Abelian sibling: namely, the analysis of the full off-shell renormalization when all inequivalent parity-preserving dimension 6 operators (classified according to [12]) are added to the power counting renormalizable action. And this constitutes precisely the subject of the present paper.

From the point of view of the EFT renormalization programme of [1], what we achieve here is to fully evaluate all the terms appearing in the renormalized action $S$ at one loop (in the relevant sector of dimension $\leq 6$ ), expressed as

$$
S_{0}=S+\hbar \Delta_{1}+\cdots
$$

At zero antifields, $\Delta_{1}$ collects one-loop gauge-invariant counterterms. The renormalized action has the same form as the original bare action $S_{0}$; in particular, it can be expanded on a basis of gauge-invariant operators (in the zero antifield sector). However, these counterterms are not enough to renormalize the theory: one must also take into account the effects of GFRs, that are implemented according to a canonical transformation with respect to the Batalin-Vilkovisky (BV) bracket associated with the gauge symmetry of the model [1]. The transformed bare action $S_{0}^{\prime}$ takes then the form

$$
S_{0}^{\prime}=S+\hbar\left[\Delta_{1}+\left(F_{1}, S\right)\right]+\cdots,
$$

where $F_{1}$ is the one loop term in the loop expansion $F(t)=\hbar t F_{1}+\cdots$ of the generator of the canonical transformation responsible for the field-antifield redefinition: $\Phi \rightarrow \Phi^{\prime}\left(\Phi, \Phi^{*}\right)$, $\Phi^{*} \rightarrow \Phi^{*^{\prime}}\left(\Phi, \Phi^{*}\right)$ on $S_{0}^{\prime}\left[\Phi^{\prime}, \Phi^{*}\right]=S_{0}\left[\Phi, \Phi^{*}\right]$. Being canonical, this transformation preserves the fundamental BV brackets $\left(\Phi^{\prime i}, \Phi_{j}^{\prime *}\right)=\delta_{j}^{i}, \quad\left(\Phi^{\prime i}, \Phi^{\prime j}\right)=\left(\Phi_{i}^{\prime *}, \Phi_{j}^{\prime *}\right)=0$, and is obtained by solving the differential equation $\dot{S}_{0}(t)=\left(F(t), S_{0}(t)\right)$ with the boundary condition $S_{0}(0)=S_{0}$, see [1]. Such canonical transformation generalizes the usual linear wave function renormalizations of the power-counting renormalizable cases. It plays a crucial and ubiquitous role in the SMEFT renormalization program, as we will show.

The paper is organized as follows. In section 2 we set up our notation and, in order to make the work self-contained we briefly review the most salient features of the $X$-formalism. Then, in sections 3 and 4 the parameterization of the one-loop UV divergences both in the $X$ - and the target (original) theory is presented and the mapping between the two theory's 
formulations derived. GFRs are studied and their form explicitly obtained in section 5, whereas the renormalization of dimension 6 gauge invariant operators in the $X$-theory is explicitly carried out in section 6 . Finally, in section 8 we describe the one-loop mixing matrix in the original theory and compare our results with the literature. Conclusions are presented in section 9. A number of technical issues are discussed in a set of appendices presented at the end of the paper: functional identities of the $X$-theory and the propagators in appendices A, B and C; the list of gauge invariant operators in appendix D; and, finally, the on-shell operator reduction relations in appendix E.

\section{Notations and conventions}

In the $X$-formalism approach of $[16,17,20]$, the tree-level vertex functional takes the form

$$
\begin{aligned}
\Gamma^{(0)}= & \int \mathrm{d}^{4} x\left[-\frac{1}{4} F^{\mu \nu} F_{\mu \nu}+\left(D^{\mu} \phi\right)^{\dagger}\left(D_{\mu} \phi\right)-\frac{M^{2}-m^{2}}{2} X_{2}^{2}-\frac{m^{2}}{2 v^{2}}\left(\phi^{\dagger} \phi-\frac{v^{2}}{2}\right)^{2}\right. \\
& -\bar{c}\left(\square+m^{2}\right) c+\frac{1}{v}\left(X_{1}+X_{2}\right)\left(\square+m^{2}\right)\left(\phi^{\dagger} \phi-\frac{v^{2}}{2}-v X_{2}\right) \\
& +\frac{z}{2} \partial^{\mu} X_{2} \partial_{\mu} X_{2}+\frac{g_{1} v}{\Lambda^{2}} X_{2}\left(D^{\mu} \phi\right)^{\dagger}\left(D_{\mu} \phi\right)+\frac{g_{2} v}{\Lambda^{2}} X_{2} F_{\mu \nu}^{2}+\frac{g_{3} v^{3}}{6 \Lambda^{2}} X_{2}^{3} \\
& +T_{1}\left(D^{\mu} \phi\right)^{\dagger}\left(D_{\mu} \phi\right)+U F_{\mu \nu}^{2}+R X_{2}^{2} \\
& +\frac{\xi b^{2}}{2}-b(\partial A+\xi e v \chi)+\bar{\omega}\left(\square \omega+\xi e^{2} v(\sigma+v) \omega\right) \\
& \left.+\bar{c}^{*}\left(\phi^{\dagger} \phi-\frac{v^{2}}{2}-v X_{2}\right)+\sigma^{*}(-e \omega \chi)+\chi^{*} e \omega(\sigma+v)\right] .
\end{aligned}
$$

In the expression above, the first line represents the action of the Abelian HK model in the $X$-formalism, where the usual scalar field $\phi \equiv \frac{1}{\sqrt{2}}\left(\phi_{0}+i \chi\right)=\frac{1}{\sqrt{2}}(\sigma+v+i \chi)$ with $v$ the vacuum expectation value (vev) is supplemented with a singlet field $X_{2}$, that provides a gauge-invariant parametrization of the physical scalar mode. Notice also that we defined $\phi_{0}=\sigma+v$ with $\sigma$ having a zero vev. The field $X_{1}$ plays instead the role of a Lagrange multiplier: when going on-shell with this field one recovers the constraint ${ }^{1}$ $X_{2} \sim \frac{1}{v}\left(\phi^{\dagger} \phi-v^{2} / 2\right)$, which once inserted back into the first line of eq. (2.1), cancels the $m^{2}$ term leaving the usual Higgs quartic potential with coefficient $\sim M^{2} / 2 v^{2}$. Hence, Green's functions in the target theory ${ }^{2}$ have to be $m^{2}$-independent, a fact that provides a very strong check of the computations, due to the ubiquitous presence of $m^{2}$ both in Feynman amplitudes as well as invariants.

${ }^{1}$ Going on-shell with $X_{1}$ yields the condition

$$
\left(\square+m^{2}\right)\left(\phi^{\dagger} \phi-\frac{v^{2}}{2}-v X_{2}\right)=0,
$$

so that the most general solution is $X_{2}=\frac{1}{v}\left(\phi^{\dagger} \phi-\frac{v^{2}}{2}\right)+\eta, \eta$ being a scalar field of mass $m$. However, in perturbation theory the correlators of the mode $\eta$ with any gauge-invariant operators vanish [16], so that one can safely set $\eta=0$.

${ }^{2}$ We define as 'target' theory the original theory defined in terms of conventional fields. 
The $X_{1,2}$-system comes together with a constraint BRST symmetry, ensuring that the number of physical degrees of freedom in the scalar sector remains unchanged in the $X$-formalism with respect to the standard formulation relying only on the field $\phi[19,20]$. More precisely, the vertex functional (2.1) is invariant under the following BRST symmetry:

$$
s X_{1}=v c ; \quad s \phi=s X_{2}=s c=0 ; \quad s \bar{c}=\phi^{\dagger} \phi-\frac{v^{2}}{2}-v X_{2} .
$$

The associated ghost and antighost fields $c, \bar{c}$ are free. The constraint BRST differential $s$ anticommutes with the (usual) gauge group BRST symmetry of the classical action after the gauge-fixing introduced in the fifth line of eq. (2.1):

$$
s A_{\mu}=\partial_{\mu} \omega ; \quad s \omega=0 ; \quad s \bar{\omega}=b ; \quad s b=0 ; \quad s \phi=i e \omega \phi .
$$

Here $\omega(\bar{\omega})$ is the U(1) ghost (antighost); the latter field is paired into a BRST doublet with the Lagrange multiplier field $b$, enforcing the usual $R_{\xi}$ gauge-fixing condition

$$
\mathscr{F}_{\xi}=\partial A+\xi e v \chi
$$

with $\xi$ the gauge fixing parameter.

The two BRST symmetries can both be lifted to the corresponding ST identities at the quantum level, provided one introduces a suitable set of so-called antifields, i.e., external sources coupled to the relevant BRST transformations that are non-linear in the quantized fields. The antifield couplings are displayed in the last line of eq. (2.1); the ST identities are instead summarized in appendix A.

The third line of eq. (2.1) contains the dimension 6 parity preserving subset of the gauge-invariant operators described in [12], modulo for the fact that we use the zero expectation value combination $\phi^{\dagger} \phi-\frac{v^{2}}{2} \sim v X_{2}$ instead of $\phi^{\dagger} \phi$. We thus see that the classical power-counting renormalizable action is supplemented in the $X$-formalism by the $X_{2}$-dependent operators ${ }^{3}$

$$
\begin{aligned}
& \mathcal{O}_{1}^{[6]}=\int \mathrm{d}^{4} x F_{\mu \nu}^{2}\left(\phi^{\dagger} \phi-\frac{v^{2}}{2}\right) \sim \int \mathrm{d}^{4} x v X_{2} F_{\mu \nu}^{2}, \\
& \mathcal{O}_{2}^{[6]}=\int \mathrm{d}^{4} x\left(\phi^{\dagger} \phi-\frac{v^{2}}{2}\right)^{3} \sim \int \mathrm{d}^{4} x v^{3} X_{2}^{3}, \\
& \mathcal{O}_{3}^{[6]}=\int \mathrm{d}^{4} x\left(\phi^{\dagger} \phi-\frac{v^{2}}{2}\right) \square\left(\phi^{\dagger} \phi-\frac{v^{2}}{2}\right) \sim \int \mathrm{d}^{4} x v^{2} X_{2} \square X_{2}, \\
& \mathcal{O}_{4}^{[6]}=\int \mathrm{d}^{4} x\left(\phi^{\dagger} \phi-\frac{v^{2}}{2}\right)\left(D^{\mu} \phi\right)^{\dagger} D_{\mu} \phi \sim \int \mathrm{d}^{4} x v X_{2}\left(D^{\mu} \phi\right)^{\dagger} D_{\mu} \phi .
\end{aligned}
$$

Notice that the operator $\mathcal{O}_{3}^{[6]}$ is special in the sense that it does not give rise in the $X$-theory to new interaction vertices: rather it modifies the propagator of the $X_{2}$-field by rescaling the $p^{2}$-term [20] (the full set of propagators of the model is summarized in appendix C). Notice also that in comparison with the conventions of $[16,17]$ we have rescaled the higher

\footnotetext{
${ }^{3}$ In the spirit of [12] we drop operators that are on-shell equivalent, i.e., that differ by terms vanishing once the classical equations of motion are imposed.
} 
dimensional coupling constants by a factor $v / \Lambda$ in order to obtain, when mapping back to the target theory, the standard $1 / \Lambda^{2}$ pre-factor for dimension 6 operators.

To maintain a detailed comparison with [1], we provide in the following some technical details.

The relevant BV bracket is the one associated with the gauge symmetry, the constraint BRST symmetry invariance being exhausted in the $X_{1}$-equation, as shown in appendix A, see eqs. (A.4) and (A.6). Next, as the gauge group is Abelian: there is no ghost antifield, since $s \omega=0$; the BRST transformation of the gauge field is linear in the quantized fields and thus there is no need to introduce the gauge antifield $A_{\mu}^{*}$ for controlling quantum corrections $^{4}$ (although algebraically one is allowed to). Also, in the $R_{\xi}$-gauge that we employ, there is no need to introduce the antifield $\bar{\omega}^{*}$, coupled to the Nakanishi-Lautrup field $b=s \bar{\omega}$ : in fact, see appendix B, the $b$-equation (B.1) and the antighost equation (B.2) imply that at the quantum level there is no dependence on the field $b$ and moreover that the antighost dependence can be reabsorbed by the antifield redefinition (B.4). On the other hand, in the formulation of [1], where one introduces both $\bar{\omega}^{*}$ and $A_{\mu}^{*}$, the antighost-dependent sector of the action is recovered from the antifield couplings $\int \mathrm{d}^{4} x\left(A_{\mu}^{*} s A^{\mu}+\chi^{*} s \chi\right)$ via a canonical transformation with fermionic generator $F=\int \mathrm{d}^{4} x \mathscr{F}_{\xi} \bar{\omega}$ (that incidentally exactly yields the antifield redefinition in eq. (B.4)). Thus, the dimension $\leq 6$ sector of $S_{0}$ is

$$
\left.\left.\sum_{k=1}^{6} S_{0}^{[k]}\right|_{A_{\mu}^{*}=\bar{\omega}^{*}=0} \equiv \Gamma^{(0)}\right|_{b=\bar{\omega}=0} .
$$

At one loop order further operators will be radiatively generated starting from $\Gamma^{(0)}$. Those operators can be however expressed in the target theory as gauge invariant polynomials in the field $\phi$, its (symmetrized) covariant derivatives, the field strength and its ordinary derivatives. This set of variables is particularly suited in order to obtain the coefficients of the one loop invariants controlling the UV divergences of the theory [7]. Additionally, some of these operators will be on-shell equivalent; the reduction to on-shell independent operators is carried out in some detail in appendix E.

Returning to eq. (2.1), we notice that the terms in the third line of eq. (2.1) respect both BRST symmetries and thus they do not violate either the $X_{1}$-equation (A.6) or the ST identity (A.1). Finally, in the fourth row we have added the external sources $T_{1}, R, U$ required to define the $X_{2}$-equation at the quantum level in the presence of additional non power-counting renormalizable interactions, see eq. (A.7).

\section{One-loop UV divergences}

In this section we will work out the parameterization of the one-loop UV divergences in the $X$-theory for all the operators giving rise to contributions to dimension 6 operators in the target theory.

${ }^{4}$ This latter fact can be easily understood since the coupling

$$
\int \mathrm{d}^{4} x A_{\mu}^{*} s A^{\mu}=\int \mathrm{d}^{4} x A_{\mu}^{*} \partial^{\mu} \omega
$$

does not generate any interaction vertex involving $A_{\mu}^{*}$, due to the aforementioned linearity of the BRST transformation of $A_{\mu}$ in the quantum fields. 
In what follows subscripts denote functional differentiation with respect to fields and external sources. Thus, amplitudes will be denoted as, e.g., $\Gamma_{\chi \chi}^{(1)}$, meaning

$$
\left.\Gamma_{\chi \chi}^{(1)} \equiv \frac{\delta^{2} \Gamma^{(1)}}{\delta \chi(-p) \delta \chi(p)}\right|_{p=0} .
$$

A bar denotes the UV divergent part of the corresponding amplitude in the Laurent expansion around $\epsilon=4-D$, with $D$ the space-time dimension. Dimensional regularization is always implied, with amplitudes evaluated by means of the packages FeynArts and FormCalc [21, 22]. As already remarked, all amplitudes will be evaluated in the Feynman $(\xi=1)$ and Landau $(\xi=0)$ gauge; this will allow to explicitly check the gauge cancellations in gauge invariant operators and in particular, as we will see, the crucial role of the GFRs in ensuring the gauge independence of ostensibly gauge invariant quantities.

Consider now the UV divergent contributions to one-loop amplitudes. They form a local functional (in the sense of formal power series) denoted by $\bar{\Gamma}^{(1)}$. Since $\bar{\Gamma}^{(1)}$ belongs to the kernel of the linearized ST operator $\mathcal{S}_{0}$ defined in eq. (A.3), i.e.,

$$
\mathcal{S}_{0}\left(\bar{\Gamma}^{(1)}\right)=0
$$

the nilpotency of $\delta_{0}$ ensures that $\bar{\Gamma}^{(1)}$ is the sum of a gauge-invariant functional $\overline{\mathscr{I}}^{(1)}$ and a cohomologically trivial contribution $\mathcal{S}_{0}\left(\bar{Y}^{(1)}\right)$ :

$$
\bar{\Gamma}^{(1)}=\overline{\mathscr{I}}_{\mathrm{gi}}^{(1)}+\delta_{0}\left(\bar{Y}^{(1)}\right)
$$

with GFRs described by the cohomologically trivial term $\delta_{0}\left(\bar{Y}^{(1)}\right)$. Eq. (3.3) bears in fact a close resemblance with eq. (1.4), as, for the model at hand, we find the identifications

$$
\Delta_{1}=-\left.\overline{\mathcal{I}}_{\mathrm{gi}}^{(1)}\right|_{b=\bar{\omega}=0} ; \quad\left(F_{1}, S\right)=-\mathcal{S}_{0}\left(\bar{Y}^{(1)}\right) .
$$

Ultimately, we are interested in the UV divergences of dimension 6 gauge invariant operators in the target theory. To identify the invariants in the $X$-theory contributing to these operators the mapping function from the $X$ - to the target theory is needed. As explained in $[16,17]$ this amounts to solving the $X_{1,2}$-equations in the $X$-theory via the replacements in eq. (A.8) and then going on-shell with $X_{1,2}$. At the one loop level it is sufficient to impose the classical equations of motions for $X_{1,2}$. The $X_{1}$-equation gives

$$
X_{2}=\frac{1}{v}\left(\phi^{\dagger} \phi-\frac{v^{2}}{2}\right)
$$

whereas the classical $X_{2}$-equation of motion yields (at zero external sources)

$$
\left(\square+m^{2}\right)\left(X_{1}+X_{2}\right)=-\left(M^{2}-m^{2}\right) X_{2}-z \square X_{2}+\frac{g_{1} v}{\Lambda^{2}}\left(D^{\mu} \phi\right)^{\dagger} D_{\mu} \phi+\frac{g_{2} v}{\Lambda^{2}} F_{\mu \nu}^{2}+\frac{g_{3} v^{3}}{2 \Lambda^{2}} X_{2}^{2} .
$$


By inserting eqs. (3.5) and (3.6) into the solutions of the $X_{1,2}$-equations (A.9a) we obtain the explicit form of the mapping for the HK model:

$$
\begin{aligned}
\bar{c}^{*} \rightarrow & -\frac{\left(M^{2}-m^{2}\right)}{v^{2}}\left(\phi^{\dagger} \phi-\frac{v^{2}}{2}\right)-\frac{z}{v^{2}} \square\left(\phi^{\dagger} \phi-\frac{v^{2}}{2}\right)+\frac{g_{1}}{\Lambda^{2}}\left(D^{\mu} \phi\right)^{\dagger} D_{\mu} \phi+\frac{g_{2}}{\Lambda^{2}} F_{\mu \nu}^{2} \\
& +\frac{g_{3}}{2 \Lambda^{2}}\left(\phi^{\dagger} \phi-\frac{v^{2}}{2}\right)^{2}, \\
\mathscr{T}_{1} \rightarrow & \frac{g_{1}}{\Lambda^{2}}\left(\phi^{\dagger} \phi-\frac{v^{2}}{2}\right) ; \quad \mathcal{U} \rightarrow \frac{g_{2}}{\Lambda^{2}}\left(\phi^{\dagger} \phi-\frac{v^{2}}{2}\right) ; \quad \mathscr{R} \rightarrow \frac{g_{3} v^{2}}{2 \Lambda^{2}}\left(\phi^{\dagger} \phi-\frac{v^{2}}{2}\right) .
\end{aligned}
$$

\section{Dimension six operators coefficients}

For computing the UV coefficients of dimension 6 gauge-invariant operators in the target theory, we need to consider, see appendix D:

1. Operators which only depend on the external sources and contribute to dimension 6 operators in the target theory again due to the mapping in eq. (3.7). They are listed in eq. (D.1), and their UV coefficients denoted by $\vartheta_{i}$ 's;

2. Mixed field-external sources gauge-invariant operators contributing to dimension 6 operators in the target theory under the mapping in eq. (3.7); these are listed in eq. (D.2j), and their UV coefficients by $\theta_{i}$ 's;

3. Dimension 6 field-dependent gauge-invariant operators that do not involve external sources; these are listed in eq. (D.3) and their UV coefficients denoted by $\lambda_{i}$ 's.

Clearly, all the associated UV coefficients $\lambda_{i}, \theta_{i}$ and $\vartheta_{i}$ will be $\xi$-independent. In order to fix them, we need to evaluate a certain number of Feynman amplitudes and derive the projections of these operators on the relevant 1-PI Green's functions. However, and as already noticed, UV divergences of the latter cannot be parameterized in terms of the $\lambda_{i}$ 's, $\theta_{i}$ 's and $\vartheta_{i}$ 's coefficients alone, since one needs to take into account contributions from GFRs. Indeed, the latter prove essential in order to ensure gauge independence of the UV coefficients of gauge invariant operators, as we will soon explicitly show.

\section{$5 \quad$ Generalized field redefinitions}

The first and most difficult step for carrying out the off-shell renormalization program is to work out the GFRs controlled by $\delta_{0}\left(\bar{Y}^{(1)}\right)$. One needs to take them into account appropriately, otherwise the renormalization of gauge invariant operators is affected by spurious contributions arising from the incorrect subtraction of UV divergences to be removed by GFRs. In particular GFRs play a crucial role in ensuring the gauge independence of the UV coefficients of gauge invariant operators, as we will explicitly show.

In the Algebraic Renormalization approach we adopt, GFRs can be written in terms of two classes of invariants as

$$
\mathcal{S}_{0} \int \mathrm{d}^{4} x\left[P(\Phi ; \zeta)\left(\sigma^{*} \sigma+\chi^{*} \chi\right)+Q(\Phi ; \zeta)\left(\sigma^{*}(\sigma+v)+\chi^{*} \chi\right)\right]
$$


with $P$ and $Q$ some local functionals ${ }^{5}$ depending on the fields (collectively denoted by $\Phi$ ) and the external sources (collectively denoted by $\zeta$ ) and $\delta_{0}$ the linearized ST operator in eq. (A.3). For convenience, we refer to these terms as $P$ - and $Q$-invariants.

In order to get a better insight on the parameterization in eq. (5.1) let us first consider the case where $P$ and $Q$ are constant. Since one has that

$$
\begin{aligned}
\delta_{0} \int \mathrm{d}^{4} x\left(\sigma^{*} \sigma+\chi^{*} \chi\right) & =\int \mathrm{d}^{4} x\left[\sigma \frac{\delta \Gamma^{(0)}}{\delta \sigma}+\chi \frac{\delta \Gamma^{(0)}}{\delta \chi}+\sigma^{*} e \chi \omega-\chi^{*} e(v+\sigma) \omega\right] \\
& \supset-\int \mathrm{d}^{4} x e v \chi^{*} \omega,
\end{aligned}
$$

the $P$-invariant is fixed in this case by the amplitude $\bar{\Gamma}_{\omega \chi^{*}}^{(1)}$. Similarly, if $P$ depends on the fields and the gauge invariant sources $\bar{c}^{*}, R, T_{1}, U$, the $P$-invariant can be fixed by looking at antifield-dependent 1-PI amplitudes. Indeed, since the antighost equation (B.2) entails that the dependence on the antighost at loops higher than one only happens via the combination $\tilde{\chi}^{*}$ in eq. (B.4), we do not need to consider antighost amplitudes and antifield-dependent ones are sufficient.

The $Q$-invariant is trickier. Let us first notice that it does not project on $\chi^{*}, \sigma^{*}$ antifield-dependent monomials:

$$
\begin{aligned}
\mathcal{S}_{0} \int \mathrm{d}^{4} x\left(\sigma^{*}(\sigma+v)+\chi^{*} \chi\right) & =\int \mathrm{d}^{4} x\left[(\sigma+v) \frac{\delta \Gamma^{(0)}}{\delta \sigma}+\chi \frac{\delta \Gamma^{(0)}}{\delta \chi}+\sigma^{*} e \chi \omega-\chi^{*} e(v+\sigma) \omega\right] \\
& \supset \int \mathrm{d}^{4} x v^{2} \bar{c}^{*}-\int \mathrm{d}^{4} x v m^{2} \sigma .
\end{aligned}
$$

However, eq. (5.3) clearly shows that it yields a contribution to $\bar{c}^{*}$ (and the $\sigma$-tadpole).

To understand the $Q$-invariant role in the renormalization of the theory, we remark that it depends only on the combination $\phi_{0}$; therefore it is useful to rewrite the counting operator in terms of $\phi, \phi^{\dagger}$, i.e.,

$$
\mathcal{S}_{0} \int \mathrm{d}^{4} x\left(\sigma^{*}(\sigma+v)+\chi^{*} \chi\right)=\mathcal{S}_{0} \int \mathrm{d}^{4} x\left(\phi \frac{\delta \Gamma^{(0)}}{\delta \phi}+\phi^{\dagger} \frac{\delta \Gamma^{(0)}}{\delta \phi^{\dagger}}\right) .
$$

Next, observe that we are only interested in the case when the right-hand side (r.h.s.) is evaluated at $X_{1,2}=0 ;{ }^{6}$ an explicit computation shows that the r.h.s. is indeed gauge-invariant (remember that we need to use the antifield $\tilde{\chi}^{*}$, as a consequence of the antighost equation):

$$
\begin{aligned}
\mathcal{S}_{0} \int \mathrm{d}^{4} x\left(\phi \frac{\delta \Gamma^{(0)}}{\delta \phi}+\phi^{\dagger} \frac{\delta \Gamma^{(0)}}{\delta \phi^{\dagger}}\right)= & \int d^{4} x\left[-2 \phi^{\dagger} D^{2} \phi-\frac{2 m^{2}}{v^{2}}\left(\phi^{\dagger} \phi-\frac{v^{2}}{2}\right) \phi^{\dagger} \phi\right. \\
& \left.-\partial^{\mu} T_{1}\left(\phi^{\dagger} D_{\mu} \phi+\text { h.c. }\right)-T_{1}\left(\phi^{\dagger} D^{2} \phi+\text { h.c. }\right)+2 \bar{c}^{*} \phi^{\dagger} \phi\right] .
\end{aligned}
$$

\footnotetext{
${ }^{5}$ We remind the reader that in EFTs field redefinitions are, in general, non-linear in the quantized fields.

${ }^{6} X_{1,2}$-amplitudes being fixed in a purely algebraic way by eq. (A.8).
} 
Notice in particular that the dependence on $\sigma^{*}, \chi^{*}$ has disappeared; as a consequence this invariant contains a combination of gauge-invariant operators that vanish on-shell. Let us now consider what happens in the power-counting renormalizable case $\left(T_{1}=0\right.$ and $z=g_{i}=0$ ). Imposing the mapping in eq. (3.7) on the r.h.s. of eq. (5.5) we obtain: ${ }^{7}$

$$
\begin{aligned}
\mathcal{S}_{0} \int \mathrm{d}^{4} x & \left(\phi \frac{\delta \Gamma^{(0)}}{\delta \phi}+\phi^{\dagger} \frac{\delta \Gamma^{(0)}}{\delta \phi^{\dagger}}\right) \\
= & -\int \mathrm{d}^{4} x\left[2 \phi^{\dagger} D^{2} \phi+\frac{2 M^{2}}{v^{2}}\left(\phi^{\dagger} \phi-\frac{v^{2}}{2}\right)^{2}-M^{2}\left(\phi^{\dagger} \phi-\frac{v^{2}}{2}\right)\right] .
\end{aligned}
$$

On the other hand, the gauge-invariant operators of the renormalizable Abelian HK model with dimension $\leq 4$ are

$$
\int \mathrm{d}^{4} x F_{\mu \nu}^{2} ; \quad \int \mathrm{d}^{4} x\left(D^{\mu} \phi\right)^{\dagger} D_{\mu} \phi ; \quad \int \mathrm{d}^{4} x\left(\phi^{\dagger} \phi-\frac{v^{2}}{2}\right) ; \quad \int \mathrm{d}^{4} x\left(\phi^{\dagger} \phi-\frac{v^{2}}{2}\right)^{2},
$$

whereas the number of physical parameters is 3 , which are usually chosen to be: the gauge coupling $e$ associated with the coefficient of the field strength squared; the mass of the vector meson $M_{A}$, which is related to the renormalization of the vev via the tadpole invariant; and, finally, the mass of the physical scalar $M$, which appears with the quartic potential invariant. The scalar kinetic covariant term is related instead to the wave function renormalization of the two-point Higgs field and as such cannot have physical effects. If we denote by $Z^{1 / 2}$ the coefficient of the corresponding invariant (5.6), the combination in the r.h.s. of that equation is exactly the one related to the wave function renormalization $\phi \rightarrow\left(1+Z^{1 / 2}\right) \phi$.

Motivated by these remarks, we choose to express all $Q$-invariants in the $X$-theory of the form

$$
\int \mathrm{d}^{4} x Q(\Phi ; \zeta) \phi^{\dagger} D^{2} \phi
$$

with $Q(\Phi ; \zeta)$ gauge-invariant, as a linear combination of gauge invariant operators and cohomologically trivial invariants of the form

$$
\mathcal{S}_{0} \int \mathrm{d}^{4} x Q(\Phi ; \zeta)\left(\sigma^{*}(\sigma+v)+\chi^{*} \chi\right) .
$$

This provides a consistent definition of the independent gauge invariant operators generalizing the corresponding set of independent physical parameters discussed in the powercounting renormalizable case.

We also notice that in the Landau gauge $(\xi=0)$ ghosts are free and the theory enjoys an exact global invariance

$$
\delta \phi=i e \alpha \phi ; \quad \delta \phi^{\dagger}=-i e \alpha \phi^{\dagger}
$$

with $\alpha$ a constant parameter. As a consequence of this rigid $U(1)$ invariance the only allowed cohomologically trivial invariants in the Landau gauge are those of the $Q$-type;

\footnotetext{
${ }^{7}$ Observe that as announced the $m^{2}$-dependence has disappeared.
} 
$P$-invariants do not arise. We will verify this property in the explicit computations that follow. On the other hand, notice that in a general gauge, $Q$ need not be gauge-invariant and both $P$ and $Q$-type invariants are required, due to the fact that the vev renormalizes differently than the fields, as is well known in the literature [23].

We now list the monomials in the expansion of $P, Q$ contributing to the projections needed to fix the coefficients of the dimension 6 operators in eqs. (D.1), (D.2j) and (D.3). Using the notation

$$
\mathscr{Z}_{1} \equiv\left(\sigma^{*} \sigma+\chi^{*} \chi\right) ; \quad \mathscr{Z}_{2} \equiv\left(\sigma^{*}(\sigma+v)+\chi^{*} \chi\right)
$$

we obtain

$$
\begin{aligned}
\bar{Y}^{(1)}=\mathcal{S}_{0} \int \mathrm{d}^{4} x[( & \left.\rho_{0}+\rho_{1} \sigma+\rho_{2} \sigma^{2}+\rho_{3} \chi^{2}+\rho_{0 T} T_{1}\right) \mathscr{Z}_{1} \\
& +\left(\tilde{\rho}_{0}+\tilde{\rho}_{1} \sigma+\tilde{\rho}_{2} \sigma^{2}+\tilde{\rho}_{3} \chi^{2}+\tilde{\rho}_{4} \sigma \chi^{2}\right. \\
& \left.\left.+\tilde{\rho}_{0 T} T_{1}+\tilde{\rho}_{0 T T} T_{1}^{2}+\tilde{\rho}_{1 T} T_{1} \sigma+\tilde{\rho}_{3 T} T_{1} \chi^{2}\right) \mathscr{Z}_{2}\right] .
\end{aligned}
$$

The different coefficients can be then evaluated by projection onto the relevant Feynman amplitudes; their values are then

$$
\begin{aligned}
\rho_{0} & =\frac{\left(1-\delta_{\xi ; 0}\right)}{8 \pi^{2} v^{2}} \frac{M_{A}^{2}}{1+z} \frac{1}{\epsilon} ; & \rho_{1} & =-\frac{\left(1-\delta_{\xi ; 0}\right)}{4 \pi^{2} v^{3}} \frac{z M_{A}^{2}}{(1+z)^{2}}, \\
\rho_{2} & =\frac{\left(1-\delta_{\xi ; 0}\right)}{8 \pi^{2} v^{4}} \frac{z(3 z-1) M_{A}^{2}}{(1+z)^{3}} \frac{1}{\epsilon} ; & \rho_{3} & =-\frac{\left(1-\delta_{\xi ; 0}\right)}{8 \pi^{2} v^{4}} \frac{z M_{A}^{2}}{(1+z)^{2}} \frac{1}{\epsilon}, \\
\rho_{0 T} & =-\frac{\left(1-\delta_{\xi ; 0}\right)}{8 \pi^{2} v^{2}} \frac{M_{A}^{2}}{(1+z)^{2}} \frac{1}{\epsilon} ; & \tilde{\rho}_{0} & =\frac{\left(1-\delta_{\xi ; 1}\right)}{16 \pi^{2} v^{2}} M_{A}^{2}, \\
\tilde{\rho}_{1} & =-\frac{\left(1-\delta_{\xi ; 1}\right)}{8 \pi^{2} v^{3}} \frac{z M_{A}^{2}}{1+z} ; & \tilde{\rho}_{2} & =\frac{\left(1-\delta_{\xi ; 1}\right)}{8 \pi^{2} v^{4}} \frac{z(z-1) M_{A}^{2}}{(1+z)^{2}}, \\
\tilde{\rho}_{3} & =\frac{(-1)^{\delta_{\xi ; 0}}}{16 \pi^{2} v^{4}} \frac{z M_{A}^{2}}{1+z} ; & \tilde{\rho}_{4} & =-(-1)^{\delta_{\xi ; 0}} \frac{z\left[3 z+(-1)^{\delta_{\xi ; 0}}\right]}{16 \pi^{2} v^{5}(1+z)^{2}} \frac{M_{A}^{2}}{\epsilon} \\
\tilde{\rho}_{0 T} & =-\frac{\left(1-\delta_{\xi ; 1}\right)}{8 \pi^{2} v^{2}} \frac{M_{A}^{2}}{\epsilon} ; & \tilde{\rho}_{0 T T} & =\frac{\left(1-\delta_{\xi ; 1}\right)}{8 \pi^{2} v^{2}} \frac{M_{A}^{2}}{\epsilon} \\
\tilde{\rho}_{1 T} & =\frac{\left(1-\delta_{\xi ; 0}\right)}{8 \pi^{2} v^{3}} \frac{z(2+z) M_{A}^{2}}{(1+z)^{2}} \frac{1}{\epsilon} ; & \tilde{\rho}_{3 T} & =0 .
\end{aligned}
$$

Notice that in Landau gauge $\bar{Y}^{(1)}$ reduces to

$$
\begin{aligned}
\left.\bar{Y}^{(1)}\right|_{\xi=0}=\mathcal{S}_{0} \int \mathrm{d}^{4} x \frac{M_{A}^{2}}{32 \pi^{2} v^{2}} \frac{1}{\epsilon}\left[2-4 T_{1}+4 T_{1}^{2}\right. \\
\left.\quad-\frac{4}{v^{2}} \frac{z}{1+z}\left(\phi^{\dagger} \phi-\frac{v^{2}}{2}\right)+\frac{2}{v^{4}} \frac{z(3 z-1)}{(1+z)^{2}}\left(\phi^{\dagger} \phi-\frac{v^{2}}{2}\right)^{2}\right] \mathscr{Z}_{2},
\end{aligned}
$$

i.e., the polynomial $Q$ is gauge-invariant, as expected; moreover, as anticipated, all $\rho$ 's coefficients vanish in this gauge. 


\subsection{GFRs in the target theory}

It is instructive to obtain the explicit form of the GFRs in the target theory at linear order in the higher dimensional couplings. For that purpose we need to apply the mapping in eq. (3.7) to $\bar{Y}^{(1)}$ retaining only the terms linear in the $g_{i}$ 's and $z$.

We remark that the coefficients in eq. (5.13) only depend on $z$. Moreover, the image of the source $T_{1}$ under the mapping is proportional to $g_{1}$ and hence from the $T_{1}$ sector we receive contributions at the linearized level only from amplitudes linear in $T_{1}$, whose coefficients need to be evaluated at $z=0$. By taking these observations into account, one easily sees that the GFRs in the target theory at linear order in the $g_{i}$ 's and $z$ couplings take the following form:

$$
\begin{aligned}
\left(\begin{array}{c}
\phi_{0}^{\prime} \\
\chi^{\prime}
\end{array}\right)= & \left\{1+\frac{M_{A}^{2}}{16 \pi^{2} v^{2}}\left[2(1-z)\left(1-\delta_{\xi ; 0}\right)+\left(1-\delta_{\xi ; 1}\right)\right.\right. \\
& -2\left[\left(1-\delta_{\xi ; 0}\right)\left(\frac{g_{1} v}{\Lambda^{2}}+\frac{2 z}{v}\right)+\left(1-\delta_{\xi ; 1}\right)\left(\frac{g_{1} v}{\Lambda^{2}}+\frac{z}{v}\right)\right] \sigma \\
& \left.\left.-\left(\frac{2 z}{v^{2}}+\frac{g_{1}}{\Lambda^{2}}\right) \sigma^{2}-\left(\frac{z}{v^{2}}+\frac{g_{1}}{\Lambda^{2}}\right) \chi^{2}-\frac{z}{v^{3}} \sigma \chi^{2}+\cdots\right]\right\} \frac{1}{\epsilon}\left(\begin{array}{l}
\sigma \\
\chi
\end{array}\right) \\
& +\left\{1+\frac{M_{A}^{2}}{16 \pi^{2} v^{2}}\left[1-\delta_{\xi ; 1}-2\left(1-\delta_{\xi ; 1}\right)\left(\frac{z}{v}+\frac{g_{1} v}{\Lambda^{2}}\right) \sigma-\frac{z}{v^{3}} \sigma \chi^{2}\right.\right. \\
& \left.\left.-\left(1-\delta_{\xi ; 1}\right)\left(\frac{2 z}{v^{2}}+\frac{g_{1}}{\Lambda^{2}}\right) \sigma^{2}-\left[(-1)^{1+\delta_{\xi ; 0}} \frac{z}{v^{2}}+\left(1-\delta_{\xi ; 1}\right) \frac{g_{1}}{\Lambda^{2}}\right] \chi^{2}+\cdots\right]\right\} \frac{1}{\epsilon}\left(\begin{array}{l}
v \\
0
\end{array}\right),
\end{aligned}
$$

where the dots denote higher dimensional contributions that are not relevant in the one loop renormalization of the dimension 6 operators under consideration. Notice also that the contribution proportional to the constant spinor $(v, 0)^{\mathrm{T}}$ is associated with the $Q$-type invariants.

From eq. (5.15) we see that the GFRs are non-multiplicative already at one loop and in the linearized approximation.

\section{Renormalization of gauge invariant operators}

Once the cohomologically trivial sector has been fixed as in eq. (5.12) and (5.13) we can proceed to project on the one-loop amplitudes required to determine the coefficients of the invariants (D.1), (D.2j) and (D.3). As the methodology is illustrated in detail in ref. [17], we report here only the results, which have been explicitly evaluated in both Landau and Feynman gauge and found to coincide as required.

\subsection{Pure external sources invariants}

The non zero $\vartheta_{i}$ coefficients are

$$
\begin{array}{rlrl}
\vartheta_{1} & =-\frac{1}{16 \pi^{2}} \frac{M^{2}+(1+z)^{2} M_{A}^{2}}{(1+z)^{2}} \frac{1}{\epsilon} ; & \vartheta_{2} & =\frac{1}{16 \pi^{2}} \frac{-M^{4}+3(1+z)^{3} M_{A}^{2}}{(1+z)^{3}} \frac{1}{\epsilon}, \\
\vartheta_{3} & =\frac{3 M_{A}^{4}}{4 \pi^{2}} \frac{1}{\epsilon} ; & \vartheta_{4}=-\frac{1}{8 \pi^{2}} \frac{M^{2}}{(1+z)^{2}} \frac{1}{\epsilon},
\end{array}
$$




$$
\begin{aligned}
\vartheta_{5} & =\frac{1}{16 \pi^{2}} \frac{2+2 z+z^{2}}{(1+z)^{2}} \frac{1}{\epsilon} ; & \vartheta_{6} & =\frac{3}{16 \pi^{2}} \frac{M^{4}+(1+z)^{4} M_{A}^{4}}{(1+z)^{4}} \frac{1}{\epsilon}, \\
\vartheta_{7} & =\frac{9 M_{A}^{4}}{\pi^{2}} \frac{1}{\epsilon} ; & \vartheta_{8} & =\frac{1}{4 \pi^{2}(1+z)^{2}} \frac{1}{\epsilon}, \\
\vartheta_{10} & =\frac{3}{32 \pi^{2}} \frac{M^{2}+(1+z)^{3} M_{A}^{2}}{(1+z)^{3}} \frac{1}{\epsilon} ; & \vartheta_{11} & =\frac{3 M_{A}^{2}}{\pi^{2}} \frac{1}{\epsilon}, \\
\vartheta_{13} & =\frac{1}{8 \pi^{2}}\left[M_{A}^{2}+\frac{M^{2}}{(1+z)^{3}}\right] \frac{1}{\epsilon} ; & \vartheta_{15} & =\frac{1}{8 \pi^{2}(1+z)^{2}} \frac{1}{\epsilon}, \\
\vartheta_{16} & =\frac{3 M_{A}^{4}}{2 \pi^{2}} \frac{1}{\epsilon} ; & \vartheta_{17} & =\frac{1}{4 \pi^{2}} \frac{M_{A}^{2}}{(1+z)^{3}} \frac{1}{\epsilon}, \\
\vartheta_{19} & =\frac{1}{32 \pi^{2}} \frac{2+2 z+z^{2}}{(1+z)^{2}} \frac{1}{\epsilon} ; & \vartheta_{22} & =\frac{3 M_{A}^{2}}{8 \pi^{2}} \frac{1}{\epsilon}, \\
\vartheta_{23} & =\frac{1}{16 \pi^{2}}(1+z)^{2} \frac{1}{\epsilon} ; & \vartheta_{26} & =-\frac{3}{4 \pi^{2}} \frac{M_{A}^{4}}{(1+z)^{5}} \frac{1}{\epsilon}, \\
\vartheta_{27} & =\frac{144 M_{A}^{4}}{\pi^{2}} \frac{1}{\epsilon} ; & \vartheta_{29} & =-\frac{1}{8 \pi^{2}} \frac{2+3 z+3 z^{2}+z^{3}}{(1+z)^{3}}, \\
\vartheta_{32} & =-\frac{1}{8 \pi^{2}} \frac{3 M^{2}+2(1+z)^{4} M_{A}^{2}}{(1} ; & \vartheta_{36} & =-\frac{1}{4 \pi^{2}} \frac{1}{(1+z)^{3}} \frac{1}{\epsilon}, \\
\vartheta_{38} & =\frac{3 M_{A}^{4}}{2 \pi^{2}} \frac{1}{\epsilon} ; & \vartheta_{39} & =-\frac{3 M^{2}}{4 \pi^{2}(1+z)^{4}} \frac{1}{\epsilon}, \\
\vartheta_{40} & =\frac{18 M_{A}^{4}}{\pi^{2}} \frac{1}{\epsilon} ; & \vartheta_{41} & =-\frac{1}{2 \pi^{2}} \frac{1}{(1+z)^{3}} \frac{1}{\epsilon} .
\end{aligned}
$$

\subsection{Mixed field-external sources invariants}

The non zero $\theta_{i}$ coefficients are

$$
\begin{aligned}
\theta_{1}= & -\frac{1}{16 \pi^{2} v^{2}} \frac{1}{(1+z)^{3}}\left[2(1-z) M^{2}+2(1+z)^{2} M_{A}^{2}+\left(2+4 z+3 z^{2}+z^{3}\right) m^{2}\right] \frac{1}{\epsilon}, \\
\theta_{2}= & \frac{1}{8 \pi^{2} v^{2}} \frac{1}{(1+z)^{4}}\left\{(z-2) M^{4}+6(1+z)^{4} M_{A}^{4}-(1+z)\left[M^{2}+(1+z)^{3} M_{A}^{2}\right] m^{2}\right\} \frac{1}{\epsilon}, \\
\theta_{3}= & \frac{3 M_{A}^{4}}{\pi^{2} v^{2}} \frac{1}{\epsilon}, \\
\theta_{4}= & -\frac{1}{8 \pi^{2} v^{2}} \frac{1}{(1+z)^{3}}\left[(1+z) m^{2}+4 M^{2}\right] \frac{1}{\epsilon}, \\
\theta_{5}= & -\frac{1}{32 \pi^{2} v^{2}} \frac{1}{(1+z)^{2}}\left[-4 z(1+z)+4(1+z) \frac{g_{1} v^{2}}{\Lambda^{2}}+(2+z) \frac{g_{1}^{2} v^{4}}{\Lambda^{4}}\right] \frac{1}{\epsilon}, \\
\theta_{6}= & -\frac{1}{32 \pi^{2} v^{2}} \frac{1}{(1+z)^{3}}\left\{2\left(2+4 z+3 z^{2}+z^{3}\right) m^{2}\right. \\
& \left.-(1+z)\left[-4+8 z^{2}-\left(8+\frac{12 g_{1} v^{2}}{\Lambda^{2}}\right) z+\frac{3 g_{1}^{2} v^{4}}{\Lambda^{4}}\right] M_{A}^{2}+\left[4+\frac{g_{1}^{2} v^{4}}{\Lambda^{4}}(3+z)\right] M^{2}\right\},
\end{aligned}
$$




$$
\begin{aligned}
& \theta_{7}=-\frac{3 M_{A}^{2}}{8 \pi^{2} v^{2}} \frac{1}{(1+z)}\left[-4 z+\frac{g_{1} v^{2}}{\Lambda^{4}}\left(g_{1} v^{2}+4 \Lambda^{2}\right)\right] \frac{1}{\epsilon}, \\
& \theta_{8}=-\frac{g_{1}^{2} v^{2}}{16 \pi^{2} \Lambda^{4}} \frac{1}{(1+z)^{2}} \frac{1}{\epsilon}, \\
& \theta_{9}=-\frac{g_{1}}{16 \pi^{2} \Lambda^{2}} \frac{1}{(1+z)^{2}} \frac{1}{\epsilon}, \\
& \theta_{10}=-\frac{1}{32 \pi^{2} v^{2}} \frac{1}{(1+z)^{3}} \\
& \times\left[-2(1+z)^{3} M_{A}^{2}+\left(2+\frac{4 g_{1} v^{2}}{\Lambda^{2}}\right) M^{2}+\left(2+4 z+3 z^{2}+z^{3}\right) m^{2}\right] \frac{1}{\epsilon}, \\
& \theta_{11}=\frac{3 M_{A}^{2}}{4 \pi^{2} v^{2}} \frac{1}{\epsilon}, \\
& \theta_{12}=-\frac{g_{1}}{8 \pi^{2} \Lambda^{2}} \frac{1}{(1+z)^{2}} \frac{1}{\epsilon}, \\
& \theta_{13}=\frac{1}{8 \pi^{2} v^{4}} \frac{z}{(1+z)^{4}}\left[(1+z)^{2}(5+z) M_{A}^{2}+4(2-z) M^{2}+4(1+z) m^{2}\right] \frac{1}{\epsilon}, \\
& \theta_{14}=-\frac{1}{8 \pi^{2} v^{4}} \frac{1}{(1+z)^{5}}\left\{(1+z)^{2}\left(2+3 z+3 z^{2}+z^{3}\right) m^{4}+4(1+z) m^{2}\left[(1-2 z) M^{2}\right.\right. \\
& \left.\left.+(1+z)^{2} M_{A}^{2}\right]+4\left[-3(1+z)^{5} M_{A}^{4}+\left(1-4 z+z^{2}\right) M^{4}\right]\right\} \frac{1}{\epsilon}, \\
& \theta_{15}=\frac{6 M_{A}^{4}}{\pi^{2} v^{4}} \frac{1}{\epsilon}, \\
& \theta_{16}=\frac{1}{2 \pi^{2} v^{4}} \frac{1}{(1+z)^{4}}\left[2(2 z-1) M^{2}+\left(z^{2}-1\right) m^{2}\right] \frac{1}{\epsilon}, \\
& \theta_{17}=\frac{g_{2}^{2} v^{2}}{8 \pi^{2} \Lambda^{4}} \frac{1}{(1+z)^{2}} \frac{1}{\epsilon} \\
& \theta_{18}=-\frac{1}{256 \pi^{2} v^{2} \Lambda^{4}} \frac{1}{(1+z)^{3}}\left\{-64 g_{2}^{2} v^{4} M^{2}+(1+z)\left[(2+z) g_{1}^{2} v^{4}+4 g_{1} v^{2} z\left(2 g_{2} v^{2}+\Lambda^{2}\right)\right.\right. \\
& \left.+4 z\left(8 g_{2}^{2} v^{4}+4 g_{2} v^{2} \Lambda^{2}-(1+z) \Lambda^{4}\right] M_{A}^{2}\right\} \frac{1}{\epsilon}, \\
& \theta_{19}=-\frac{g_{2}^{2} v^{2}}{2 \pi^{2} \Lambda^{4}} \frac{1}{(1+z)^{2}}\left[M^{2}+2(1+z) M_{A}^{2}\right] \frac{1}{\epsilon} \text {, } \\
& \theta_{20}=\frac{g_{2}^{2} v^{2}}{4 \pi^{2} \Lambda^{4}} \frac{1}{(1+z)^{2}} \frac{1}{\epsilon}, \\
& \theta_{21}=-\frac{1}{4 \pi^{2} v^{2}} \frac{z}{(1+z)^{3}} \frac{1}{\epsilon} \\
& \theta_{22}=\frac{1}{8 \pi^{2} v^{2}} \frac{1}{(1+z)^{5}}\left[6(1-z) M^{4}+6(1+z)^{5} M_{A}^{4}+(1+z)\left(3 M^{2}+2(1+z)^{4} M_{A}^{2}\right) m^{2}\right] \frac{1}{\epsilon}, \\
& \theta_{23}=\frac{36 M_{A}^{4}}{\pi^{2} v^{2}} \frac{1}{\epsilon} \\
& \theta_{24}=\frac{1}{\pi^{2} v^{2}} \frac{1}{(1+z)^{3}} \frac{1}{\epsilon},
\end{aligned}
$$




$$
\begin{aligned}
\theta_{25} & =\frac{1}{8 \pi^{2} v^{2}} \frac{1}{(1+z)^{4}}\left[(2-4 z) M^{2}+2(1+z)^{2} M_{A}^{2}+\left(2+5 z+6 z^{2}+4 z^{3}+z^{4}\right) m^{2}\right] \frac{1}{\epsilon} \\
\theta_{27} & =\frac{1}{4 \pi^{2} v^{2}} \frac{(1-z)}{(1+z)^{3}} \frac{1}{\epsilon} \\
\theta_{28} & =\frac{6 M_{A}^{4}}{\pi^{2} v^{2}} \frac{1}{\epsilon} \\
\theta_{29} & =\frac{1}{4 \pi^{2} v^{2}} \frac{1}{(1+z)^{4}}\left[2(2-z) M^{2}+(1+z) m^{2}\right] \frac{1}{\epsilon}
\end{aligned}
$$

\subsection{Gauge invariants depending only on the fields}

The non zero $\lambda_{i}$ coefficients are

$$
\begin{aligned}
& \lambda_{1}=\frac{1}{16 \pi^{2} v^{2}} \frac{1}{(1+z)^{3}}\left\{(1+z)\left[M^{2}+(1+z)^{2} M_{A}^{2}\right] m^{2}+2\left[M^{4}+3(1+z)^{3} M_{A}^{4}\right]\right\} \frac{1}{\epsilon}, \\
& \lambda_{2}=\frac{1}{32 \pi^{2} v^{4}} \frac{1}{(1+z)^{4}}\left\{4(1-2 z) M^{4}+4 m^{2} M_{A}^{2}(1+z)^{3}+12(1+z)^{4} M_{A}^{4}\right. \\
& \left.+4 m^{2} M^{2}\left(1-z^{2}\right)+(1+z)^{2}\left(2+2 z+z^{2}\right) m^{4}\right\} \frac{1}{\epsilon}, \\
& \lambda_{3}=-\frac{1}{16 \pi^{2} v^{6}} \frac{z}{(1+z)^{5}}\left\{8(1-z) M^{4}+2(1+z)^{2} m^{4}\right. \\
& \left.+(1+z)\left[4(2-z) M^{2}+(1+z)^{2}(5+z) M_{A}^{2}\right] m^{2}\right\} \frac{1}{\epsilon}, \\
& \lambda_{4}=-\frac{1}{32 \pi^{2} v^{2}} \frac{1}{(1+z)^{2}} \\
& \times\left\{(1+z)\left[16+4 z+\frac{3 g_{1}^{2} v^{4}}{\Lambda^{4}}+12 \frac{g_{1} v^{2}}{\Lambda^{2}}\right] M_{A}^{2}+\frac{g_{1} v^{2}}{\Lambda^{2}}\left(4-\frac{g_{1} v^{2}}{\Lambda^{2}}\right) M^{2}\right\} \frac{1}{\epsilon}, \\
& \lambda_{5}=\frac{g_{1}^{2} v^{2}}{192 \pi^{2} \Lambda^{4}} \frac{1}{(1+z)} \frac{1}{\epsilon}, \\
& \lambda_{6}=\frac{1}{64 \pi^{2} v^{4}} \frac{1}{(1+z)^{3}}\left\{\left[4 z+4(1-3 z) \frac{g_{1} v^{2}}{\Lambda^{2}}+(1+z) \frac{g_{1}^{2} v^{4}}{\Lambda^{4}}\right] M^{2}\right. \\
& \left.+(1+z)^{2}\left(4 z-12 \frac{g_{1} v^{2}}{\Lambda^{2}}-3 \frac{g_{1}^{2} v^{4}}{\Lambda^{4}}\right) M_{A}^{2}+4(1+z) \frac{g_{1} v^{2}}{\Lambda^{2}} m^{2}\right\} \frac{1}{\epsilon}, \\
& \lambda_{7}=\frac{1}{32 \pi^{2} v^{4}} \frac{1}{(1+z)^{3}}\left\{\left[4 z-4(1+z) \frac{g_{1} v^{2}}{\Lambda^{2}}+(5+z) \frac{g_{1}^{2} v^{4}}{\Lambda^{4}}\right] M^{2}\right. \\
& +3(1+z)\left[4 z(3+z)-4(3+z) \frac{g_{1} v^{2}}{\Lambda^{2}}-(5+3 z) \frac{g_{1}^{2} v^{4}}{\Lambda^{4}}\right] M_{A}^{2} \\
& \left.+(1+z)\left[-4 z(1+z)+4(1+z) \frac{g_{1} v^{2}}{\Lambda^{2}}+(2+z) \frac{g_{1}^{2} v^{4}}{\Lambda^{4}}\right] m^{2}\right\} \frac{1}{\epsilon}, \\
& \lambda_{8}=-\frac{1}{192 \pi^{2} v^{2}} \frac{1}{(1+z)^{2}}\left\{48 \frac{g_{2}^{2} v^{4}}{\Lambda^{4}} M^{2}\right. \\
& \left.+(1+z)\left[2+48\left(\frac{g_{2} v^{2}}{\Lambda^{2}}+\frac{g_{2}^{2} v^{4}}{\Lambda^{4}}\right)+2 g_{1} \frac{v^{2}}{\Lambda^{2}}+24 g_{1} g_{2} \frac{v^{4}}{\Lambda^{4}}+g_{1}^{2} \frac{v^{4}}{\Lambda^{4}}\right] M_{A}^{2}\right\} \frac{1}{\epsilon}, \\
& \lambda_{9}=-\frac{g_{2}^{2} v^{2}}{6 \pi^{2} \Lambda^{4}} \frac{1}{(1+z)} \frac{1}{\epsilon}
\end{aligned}
$$




$$
\begin{aligned}
\lambda_{10}= & \frac{1}{128 \pi^{2} v^{2} \Lambda^{2}} \frac{1}{(1+z)^{3}}\left\{-64 \frac{g_{2}^{2} v^{2}}{\Lambda^{2}} M^{2}+(1+z)\left[g_{1}^{3} \frac{v^{4}}{\Lambda^{4}}-4 \frac{g_{1}^{2} v^{2}}{\Lambda^{2}}\right.\right. \\
& \left.\left.-8(2+z) g_{1} g_{2} \frac{v^{2}}{\Lambda^{2}}-16(2+z)\left(2 g_{2}^{2} \frac{v^{2}}{\Lambda^{2}}+g_{2}\right)\right] M_{A}^{2}-16(1+z) \frac{g_{2}^{2} v^{2}}{\Lambda^{2}} m^{2}\right\} \frac{1}{\epsilon}
\end{aligned}
$$

\section{Mapping to the target theory}

The UV coefficients in the target theory $\tilde{\lambda}_{i}$ can be obtained by: applying the mapping in eq. (3.7) to the invariants in eqs. (D.1) and (D.2j); combining the projections with the operators in (D.3); and, finally, using the results (6.1), (6.2) and (6.3). Notice that for these coefficients all $\mathrm{m}^{2}$-dependent contributions must cancel out; we have checked this explicitly.

The coefficients so obtained represents the complete one-loop renormalizations of the corresponding operators; in particular, no linearized approximation in the higher dimensional couplings $g_{i}$ 's has been made so far. However, as the resulting general expressions are rather lengthy, we report below the non zero coefficients $\tilde{\lambda}_{i}$ at linear order in the $g_{i}$ couplings:

$$
\begin{aligned}
& \tilde{\lambda}_{1} \sim-\frac{1}{16 \pi^{2} v^{2}}\left[8 z M_{A}^{4}+\left(M^{4}-3 M_{A}^{4}\right) \frac{g_{1} v^{2}}{\Lambda^{2}}-12 M_{A}^{4} \frac{g_{2} v^{2}}{\Lambda^{2}}+M^{2} g_{3} \frac{v^{4}}{\Lambda^{2}}\right] \frac{1}{\epsilon} \\
& \tilde{\lambda}_{2} \sim-\frac{1}{32 \pi^{2} v^{4}}\left[\left(4 M^{2} M_{A}^{2}+42 M^{4}\right) z+4\left(3 M^{4}+M^{2} M_{A}^{2}-6 M_{A}^{4}\right) \frac{g_{1} v^{2}}{\Lambda^{2}}\right. \\
&\left.-96 \frac{g_{2} v^{2}}{\Lambda^{2}} M_{A}^{4}+\left(11 M^{2}+M_{A}^{2}\right) \frac{g_{3} v^{4}}{\Lambda^{2}}\right] \frac{1}{\epsilon}, \\
& \tilde{\lambda}_{3} \sim-\frac{1}{16 \pi^{2} v^{6}}\left[z M^{2}\left(18 M^{2}+5 M_{A}^{2}\right)+2\left(5 M^{4}+2 M^{2} M_{A}^{2}-6 M_{A}^{4}\right) \frac{g_{1} v^{2}}{\Lambda^{2}}\right. \\
&\left.-48 M_{A}^{4} \frac{g_{2} v^{2}}{\Lambda^{2}}+\left(8 M^{2}+M_{A}^{2}\right) \frac{g_{3} v^{4}}{\Lambda^{2}}\right] \frac{1}{\epsilon}, \\
& \tilde{\lambda}_{4} \sim \frac{1}{16 \pi^{2} v^{2}}\left[6 z M_{A}^{2}-\left(3 M^{2}+7 M_{A}^{2}\right) \frac{g_{1} v^{2}}{\Lambda^{2}}\right] \frac{1}{\epsilon}, \\
& \tilde{\lambda}_{6} \sim \frac{1}{16 \pi^{2} v^{4}}\left[z\left(5 M^{2}+3 M_{A}^{2}\right)-2 M_{A}^{2} \frac{g_{1} v^{2}}{\Lambda^{2}}+12 M_{A}^{2} \frac{g_{2} v^{2}}{\Lambda^{2}}\right] \frac{1}{\epsilon}, \\
& \tilde{\lambda}_{7} \sim \frac{1}{8 \pi^{2} v^{4}}\left(4 M^{2}+11 M_{A}^{2}\right)\left(z-\frac{g_{1} v^{2}}{\Lambda^{2}}\right) \frac{1}{\epsilon}, \\
& \tilde{\lambda}_{8} \sim-\frac{1}{96 \pi^{2} v^{2}}\left[6 \frac{g_{2} v^{2}}{\Lambda^{2}}\left(M^{2}+5 M_{A}^{2}\right)+M_{A}^{2}\left(-z+\frac{g_{1} v^{2}}{\Lambda^{2}}\right)\right] \frac{1}{\epsilon}, \\
& \tilde{\lambda}_{10} \sim-\frac{1}{8 \pi^{2} v^{4}} \frac{g_{2} v^{2}}{\Lambda^{2}}\left(2 M^{2}+M_{A}^{2}\right) \frac{1}{\epsilon} .
\end{aligned}
$$

We hasten to emphasize that GFRs do contribute also at the linearized level, as has been discussed in detail in section 5.1. Failure to take their contributions into account would lead to an erroneous determination of the coefficients in eq. (7.1).

The $g_{i}$ 's, $z$ contributions to the $\beta$ functions

$$
\beta_{i}=(4 \pi)^{2} \frac{d}{d \log \mu} \tilde{\lambda}_{i}
$$


can then be easily determined from eq. (7.1), leading to:

$$
\begin{aligned}
\beta_{1} \supset & -\frac{1}{v^{2}}\left[8 z M_{A}^{4}+\left(M^{4}-3 M_{A}^{4}\right) \frac{g_{1} v^{2}}{\Lambda^{2}}-12 M_{A}^{4} \frac{g_{2} v^{2}}{\Lambda^{2}}+M^{2} g_{3} \frac{v^{4}}{\Lambda^{2}}\right], \\
\beta_{2} \supset & -\frac{1}{v^{4}}\left[\left(4 M^{2} M_{A}^{2}+42 M^{4}\right) z+4\left(3 M^{4}+M^{2} M_{A}^{2}-6 M_{A}^{4}\right) \frac{g_{1} v^{2}}{\Lambda^{2}}\right. \\
& \left.-96 \frac{g_{2} v^{2}}{\Lambda^{2}} M_{A}^{4}+\left(11 M^{2}+M_{A}^{2}\right) \frac{g_{3} v^{4}}{\Lambda^{2}}\right], \\
\beta_{3} \supset & -\frac{3}{v^{6}}\left[z M^{2}\left(18 M^{2}+5 M_{A}^{2}\right)+2\left(5 M^{4}+2 M^{2} M_{A}^{2}-6 M_{A}^{4}\right) \frac{g_{1} v^{2}}{\Lambda^{2}}\right. \\
& \left.-48 M_{A}^{4} \frac{g_{2} v^{2}}{\Lambda^{2}}+\left(8 M^{2}+M_{A}^{2}\right) \frac{g_{3} v^{4}}{\Lambda^{2}}\right], \\
\beta_{4} \supset & \frac{1}{v^{2}}\left[6 z M_{A}^{2}-\left(3 M^{2}+7 M_{A}^{2}\right) \frac{g_{1} v^{2}}{\Lambda^{2}}\right], \\
\beta_{6} \sim & \frac{2}{v^{4}}\left[z\left(5 M^{2}+3 M_{A}^{2}\right)-2 M_{A}^{2} \frac{g_{1} v^{2}}{\Lambda^{2}}+12 M_{A}^{2} \frac{g_{2} v^{2}}{\Lambda^{2}}\right], \\
\beta_{7} \supset & \frac{4}{v^{4}}\left(4 M^{2}+11 M_{A}^{2}\right)\left(z-\frac{g_{1} v^{2}}{\Lambda^{2}}\right), \\
\beta_{8} \supset & -\frac{1}{6 v^{2}}\left[6 \frac{g_{2} v^{2}}{\Lambda^{2}}\left(M^{2}+5 M_{A}^{2}\right)+M_{A}^{2}\left(-z+\frac{g_{1} v^{2}}{\Lambda^{2}}\right)\right], \\
\beta_{10} \sim & -\frac{4}{v^{4}} \frac{g_{2} v^{2}}{\Lambda^{2}}\left(2 M^{2}+M_{A}^{2}\right) .
\end{aligned}
$$

\section{One-loop mixing matrices}

We are now in a position to compare our results with those in the literature $[24,25]$. By inspecting eq. (7.1) we obtain the mixing matrix represented in table 1. We find agreement with the results of $[24,25]$ with the exception of the mixing of $\phi^{4} D^{2}$ operators with $F^{2} \phi^{2}$. More specifically, a closer inspection of eq. (7.1) shows that the operator

$$
\mathscr{I}_{7}=\int \mathrm{d}^{4} x\left(\phi^{\dagger} \phi-\frac{v^{2}}{2}\right)\left(D^{\mu} \phi\right)^{\dagger} D_{\mu} \phi
$$

respects the mixing pattern derived in [24, 25], whereas the operator

$$
\mathscr{I}_{6}=\int \mathrm{d}^{4} x\left(\phi^{\dagger} \phi-\frac{v^{2}}{2}\right)\left(\phi^{\dagger} D^{2} \phi+\text { h.c. }\right)
$$

does not, since it mixes with

$$
\mathscr{I}_{10}=\int \mathrm{d}^{4} x F_{\mu \nu}^{2}\left(\phi^{\dagger} \phi-\frac{v^{2}}{2}\right) .
$$

There is an elegant cohomological interpretation of this result. One can find $\delta_{0^{-}}$ invariant combinations of gauge invariant operators that do not depend on the antifields, in very much the same way as in eq. (5.14). Notice that these invariants depend on $\sigma, \chi$ 


\begin{tabular}{|c|c|c|c|}
\hline & $F^{2} \phi^{2}$ & $\phi^{4} D^{2}$ & $\phi^{6}$ \\
\hline \multicolumn{4}{|l|}{$F^{2} \phi^{2}$} \\
\hline$\phi^{4} D^{2}$ & $\times$ & & \\
\hline$\phi^{6}$ & & & \\
\hline
\end{tabular}

Table 1. One-loop operator mixing matrix in the Abelian HK model. Shaded entries denote a vanishing coefficient. The $\times$ indicates an entry that should vanish according to the non-renormalization theorem of $[24,25]$ but that does not given the coefficients in eq. (7.1).

only via $\phi$ and they are generated by $\mathscr{Z}_{2}$ (now to be understood in the target theory). In particular one finds

$$
\begin{aligned}
\mathcal{S}_{0} \int \mathrm{d}^{4} x \mathscr{Z}_{2} & =\int \mathrm{d}^{4} x\left(\phi \frac{\delta S}{\delta \phi}+\phi^{\dagger} \frac{\delta S}{\delta \phi^{\dagger}}\right) \\
& =\int \mathrm{d}^{4} x\left[-\left(D^{2} \phi\right)^{\dagger} \phi-\phi^{\dagger} D^{2} \phi-2 \frac{M^{2}}{v^{2}}\left(\phi^{\dagger} \phi-\frac{v^{2}}{2}\right) \phi^{\dagger} \phi\right],
\end{aligned}
$$

which is gauge-invariant. Thus any invariant of the form

$$
\mathcal{S}_{0} \int \mathrm{d}^{4} x Q\left(\phi, \phi^{\dagger}, A_{\mu}\right) \mathscr{E}_{2},
$$

is gauge invariant if $Q$ is a gauge-invariant polynomial. Being cohomologically trivial, the above family of invariants can be added order by order in the loop expansion without changing the physical observables of the theory. Intuitively the simultaneous variation of the coefficients of the operators entering in the invariants (8.5) cannot affect the physics since the variation is proportional to the equations of motion. This is an example of the aforementioned fact that the mixing between gauge-invariant and alien operators (which are cohomologically trivial with respect to the linearized ST operator) can be made to vanish by a suitable basis choice in the space of local operators [2-6].

This means that there is the freedom to replace the invariant $\mathscr{I}_{6}$ with the linear combination of $\mathscr{I}_{2}$ and $\mathscr{I}_{3}$ in eq. (E.16) up to a cohomologically trivial $\mathcal{S}_{0}$-invariant. This transformation induces the following shift on the space of the $\tilde{\lambda}$ 's parameters:

$$
\tilde{\lambda}_{2} \rightarrow \widetilde{\lambda}_{2}-M^{2} \widetilde{\lambda}_{6} ; \quad \widetilde{\lambda}_{3} \rightarrow \widetilde{\lambda}_{3}-\frac{2 M^{2}}{v^{2}} \widetilde{\lambda}_{6} .
$$

For this new basis then, the non-renormalization theorem of $[24,25]$ hold true.

Notice that in the analysis of the mixing of dimension 6 operators carried out in [24, 25], the on-shellness of the external physical scalar particles has been explicitly assumed. This meant in particular that (for external particles)

$$
\left.\square \sigma\right|_{\text {on-shell }} \sim M^{2} \sigma,
$$

This choice, however, makes compulsory the use of a basis for dimension 6 operators in which $D^{2} \phi$ has been eliminated through its equation of motion, since only in this case the linearized approximation gives back eq. (8.7). 


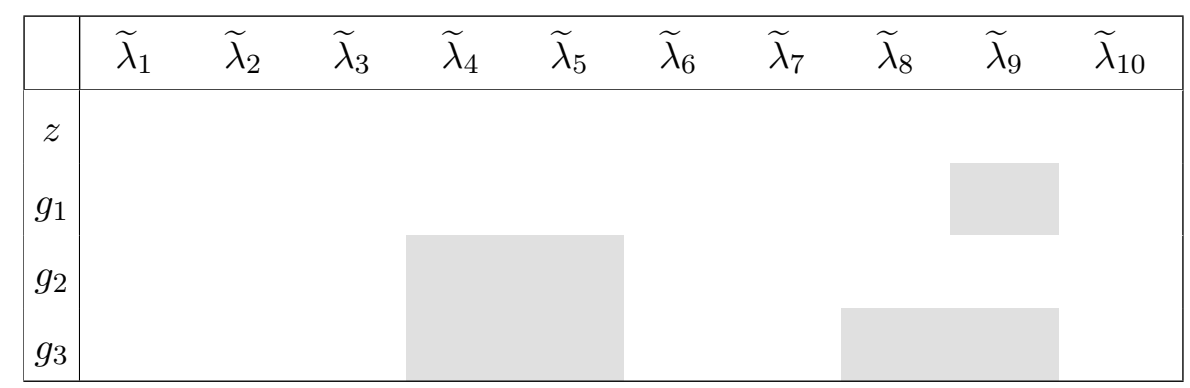

Table 2. Dependence of the $\widetilde{\lambda}_{i}$ 's on the higher dimensional coupling constants. Shaded entries denote that the dependence of the $\tilde{\lambda}_{i}$ parameter on the corresponding coupling constant vanishes.

A related question is whether in the recursive subtraction procedure one can indeed limit himself only to the on-shell inequivalent operators chosen at tree-level (in the present case, in the given dimension 6 layer). The answer is in the negative. Neglecting onshell vanishing operators at order $n$ in the loop expansion hampers the subtraction of the next order UV divergences by local counterterms, thus destroying the consistency of the procedure. Both the on-shell inequivalent invariants (in the chosen basis) and the $\delta_{0}$-exact operators have to be taken into account beyond the leading order.

In order to study the one-loop amplitudes dependence on the $g_{i}$ 's and $z$ beyond the single higher-dimensional operator insertion approximation commonly used in the literature, we have reported in table 2 the dependence of the (shifted) $\tilde{\lambda}$ 's coefficients on the $g_{i}$ 's and $z$, based on the full one loop computation carried out in the present paper.

The vanishing entries in table 2 can be partially understood in terms of the underlying amplitudes decomposition made transparent by the $X$-formalism. As explained above, the $\widetilde{\lambda}$ 's are a linear combination of the $\lambda$ 's coefficients multiplying gauge invariant operators which are independent from external sources of the $X$-theory, and of the coefficients $\vartheta, \theta$ 's associated with invariants involving external sources insertions (the UV behaviour of which is more constrained than that of the fields). In particular, we find for the relevant operators in table 2 :

$$
\tilde{\lambda}_{4}=\lambda_{4}+\frac{g_{1} \vartheta_{1}}{\Lambda^{2}} ; \quad \tilde{\lambda}_{5}=\lambda_{5} ; \quad \tilde{\lambda}_{8}=\lambda_{8}+\frac{g_{2} \vartheta_{1}}{\Lambda^{2}} ; \quad \tilde{\lambda}_{9}=\lambda_{9} .
$$

The $\vartheta_{1}$-terms can be neglected: they can only induce a $z$-dependence and thus do not contribute to the cancellations in table 2 . Hence, the problem is reduced to the determination of the $g_{i}$ 's dependence of the $\lambda$ 's coefficients in the $X$-theory. One immediately sees that these coefficients cannot depend on $g_{3}$ since this is a trilinear vertex in $X_{2}$ that does not contribute to the 1-PI amplitudes of the starting theory at one loop. Thus, the last row of table 2 must hold, as the only possible dependence on $g_{3}$ at one loop arises from the mapping to the target theory in eq. (3.7) and therefore governed by external amplitudes involving $\bar{c}^{*}$ and/or $R$ external sources, which do not enter in eq. (8.8).

The remaining three forbidden dependences just seem to be an accidental consequence of the one-loop Feynman diagrams; as a result, cancellation patterns do not seem to lend themselves to an easy generalization to higher orders. 


\section{Conclusions}

In the present paper we have completed the investigation of the one-loop off-shell renormalization of the Abelian Higgs-Kibble model supplemented at tree-level with all dimension 6 parity preserving on-shell inequivalent gauge-invariant operators. This was the last step towards the analysis of the $\mathrm{SU}(2) \times \mathrm{U}(1)$ case.

We have shown that the $X$-theory formalism provides an effective way to work out the relevant GFRs, which in turn are found to have an ubiquitous effect on the one-loop UV coefficients of dimension 6 operators. In fact, since the GFRs are non linear and even non polynomial in the fields, it is advantageous to employ cohomological tools in order to disentangle the UV coefficients of the gauge-invariant operators from the spurious (and gauge-dependent) contributions associated with GFRs.

We have provided a full one-loop computation going beyond the customary linearized approximation in the higher dimensional couplings. All coefficients have been evaluated both in Feynman and in Landau gauge and the gauge independence of the UV coefficients of the gauge invariant operators explicitly checked. As expected, it does not hold unless the effects of GFRs are properly accounted for.

We find that the pattern of operator mixing cancellations studied in the previous literature only holds off-shell if an appropriate choice of the on-shell equivalent operators is made. This can be traced back to the freedom of adding cohomologically trivial combinations of gauge-invariant operators at one loop order, thus selecting a particular basis of gauge-invariant on-shell inequivalent operators.

Application of the method presented to the SMEFT is currently under investigation.

\section{A Functional identities in the $X$-theory}

\section{A.1 ST identities}

The ST identity (also known as the master equation in the BV approach) associated to the gauge group BRST symmetry reads

$$
\mathcal{S}(\Gamma)=\int \mathrm{d}^{4} x\left[\partial_{\mu} \omega \frac{\delta \Gamma}{\delta A_{\mu}}+\frac{\delta \Gamma}{\delta \sigma^{*}} \frac{\delta \Gamma}{\delta \sigma}+\frac{\delta \Gamma}{\delta \chi^{*}} \frac{\delta \Gamma}{\delta \chi}+b \frac{\delta \Gamma}{\delta \bar{\omega}}\right]=0,
$$

or, at order $n$ in the loop expansion,

$$
\mathcal{S}(\Gamma)^{(n)}=\mathcal{S}_{0}\left(\Gamma^{(n)}\right)+\sum_{j=1}^{n-1}\left(\frac{\delta \Gamma^{(j)}}{\delta \sigma^{*}} \frac{\delta \Gamma^{(n-j)}}{\delta \sigma}+\frac{\delta \Gamma^{(j)}}{\delta \chi^{*}} \frac{\delta \Gamma^{(n-j)}}{\delta \chi}\right)=0,
$$

where $\mathcal{S}_{0}$ is the linearized ST operator:

$$
\begin{aligned}
\delta_{0}\left(\Gamma^{(n)}\right)=\int \mathrm{d}^{4} x\left[\partial_{\mu} \omega \frac{\delta \Gamma^{(n)}}{\delta A_{\mu}}+e \omega(\sigma+v) \frac{\delta \Gamma^{(n)}}{\delta \chi}-e \omega \chi \frac{\delta \Gamma^{(n)}}{\delta \sigma}+b \frac{\delta \Gamma^{(n)}}{\delta \bar{\omega}}\right. \\
\left.+\frac{\delta \Gamma^{(0)}}{\delta \sigma} \frac{\delta \Gamma^{(n)}}{\delta \sigma^{*}}+\frac{\delta \Gamma^{(0)}}{\delta \chi} \frac{\delta \Gamma^{(n)}}{\delta \chi^{*}}\right] \\
=s \Gamma^{(n)}+\int \mathrm{d}^{4} x\left[\frac{\delta \Gamma^{(0)}}{\delta \sigma} \frac{\delta \Gamma^{(n)}}{\delta \sigma^{*}}+\frac{\delta \Gamma^{(0)}}{\delta \chi} \frac{\delta \Gamma^{(n)}}{\delta \chi^{*}}\right] .
\end{aligned}
$$


$\delta_{0}$ maps the antifields $\sigma^{*}, \chi^{*}$ into the equations of motion of the fields $\sigma, \chi$, while it acts on the fields as the BRST operator $s$. Notice that, as explained before, we do not introduce an antifield for the gauge field $A_{\mu}$ since in the Abelian case treated here the gauge BRST transformation is linear.

The ST identity for the constraint BRST symmetry is

$$
\mathcal{S}_{C}(\Gamma) \equiv \int \mathrm{d}^{4} x\left[v c \frac{\delta \Gamma}{\delta X_{1}}+\frac{\delta \Gamma}{\delta \bar{c}^{*}} \frac{\delta \Gamma}{\delta \bar{c}}\right]=\int \mathrm{d}^{4} x\left[v c \frac{\delta \Gamma}{\delta X_{1}}-\left(\square+m^{2}\right) c \frac{\delta \Gamma}{\delta \bar{c}^{*}}\right]=0,
$$

where in the latter equality we have used the fact that both the ghost $c$ and the antighost $\bar{c}$ are free:

$$
\frac{\delta \Gamma}{\delta \bar{c}}=-\left(\square+m^{2}\right) c ; \quad \frac{\delta \Gamma}{\delta c}=\left(\square+m^{2}\right) \bar{c} .
$$

\section{A.2 $X_{1,2}$-equations}

By using eq. (A.5) one sees that eq. (A.4) reduces to the $X_{1}$-equation of motion

$$
\frac{\delta \Gamma}{\delta X_{1}}=\frac{1}{v}\left(\square+m^{2}\right) \frac{\delta \Gamma}{\delta \bar{c}^{*}} .
$$

Notice that this equation stays the same irrespectively of the presence of higher-dimensional gauge invariant operators added to the power-counting renormalizable action.

The $X_{2}$-equation is in turn given by

$$
\begin{aligned}
\frac{\delta \Gamma}{\delta X_{2}}= & \frac{1}{v}\left(\square+m^{2}\right) \frac{\delta \Gamma}{\delta \bar{c}^{*}}+\frac{g_{1} v}{\Lambda^{2}} \frac{\delta \Gamma}{\delta T_{1}}+\frac{g_{2} v}{\Lambda^{2}} \frac{\delta \Gamma}{\delta U}+\frac{g_{3} v^{3}}{2 \Lambda^{2}} \frac{\delta \Gamma}{\delta R}-\left(\square+m^{2}\right) X_{1} \\
& -\left[(1+z) \square+M^{2}\right] X_{2}-v \bar{c}^{*} .
\end{aligned}
$$

\section{A.3 Solving the $X_{1,2}$-equations}

At order $n, n \geq 1$ in the loop expansion the $X_{1,2}$-equations reduce to

$$
\begin{aligned}
& \frac{\delta \Gamma^{(n)}}{\delta X_{1}}=\frac{1}{v}\left(\square+m^{2}\right) \frac{\delta \Gamma^{(n)}}{\delta \bar{c}^{*}}, \\
& \frac{\delta \Gamma^{(n)}}{\delta X_{2}}=\frac{1}{v}\left(\square+m^{2}\right) \frac{\delta \Gamma^{(n)}}{\delta \bar{c}^{*}}+\frac{g_{1} v}{\Lambda^{2}} \frac{\delta \Gamma^{(n)}}{\delta T_{1}}+\frac{g_{2} v}{\Lambda^{2}} \frac{\delta \Gamma^{(n)}}{\delta U}+\frac{g_{3} v^{3}}{2 \Lambda^{2}} \frac{\delta \Gamma^{(n)}}{\delta R} .
\end{aligned}
$$

By using the chain rule for functional differentiation it is straightforward to see that eqs. (A.8) entail that $\Gamma^{(n)}$ only depends on the combinations:

$$
\begin{aligned}
\bar{c}^{*} & =\bar{c}^{*}+\frac{1}{v}\left(\square+m^{2}\right)\left(X_{1}+X_{2}\right) ; & \mathscr{T}_{1} & =T_{1}+\frac{g_{1} v}{\Lambda^{2}} X_{2}, \\
\mathcal{U} & =U+\frac{g_{2} v}{\Lambda^{2}} X_{2} ; & \mathscr{R} & =R+\frac{g_{3} v^{3}}{2 \Lambda^{2}} X_{2} .
\end{aligned}
$$

\section{B The $b$ - and the gauge ghost equation}

The set of the functional identities holding in the $X$-formulation of the Abelian HK model is completed by: 
- The b-equation:

$$
\frac{\delta \Gamma}{\delta b}=\xi b-\partial A-\xi e v \chi
$$

- The antighost equation:

$$
\frac{\delta \Gamma}{\delta \bar{\omega}}=\square \omega+\xi e v \frac{\delta \Gamma}{\delta \chi^{*}} .
$$

At orders $n \geq 1$ the $b$ - and the antighost equations imply

$$
\frac{\delta \Gamma^{(n)}}{\delta b}=0 ; \quad \frac{\delta \Gamma^{(n)}}{\delta \bar{\omega}}=\xi e v \frac{\delta \Gamma^{(n)}}{\delta \chi^{*}},
$$

so that at higher orders the vertex functional does not depend on the Nakanishi-Lautrup field $b$ and the dependence on the antighost is only via the combination

$$
\widetilde{\chi}^{*} \equiv \chi^{*}+\xi e v \bar{\omega}
$$

\section{Propagators}

\section{C.1 The $X-\sigma$ sector}

Diagonalization of the quadratic part of the action in this sector is achieved by setting

$$
\sigma=\sigma^{\prime}+X_{1}+X_{2}
$$

Then one has

$$
\Delta_{\sigma^{\prime} \sigma^{\prime}}=\frac{i}{p^{2}-m^{2}} ; \quad \Delta_{X_{1} X_{1}}=-\frac{i}{p^{2}-m^{2}} ; \quad \Delta_{X_{2} X_{2}}=\frac{i}{(1+z) p^{2}-M^{2}} .
$$

Several comments are in order here. At $g_{1}, g_{2}, g_{3}=0$ no higher dimensional interactions vertices are present. However, the model is still non power-counting renormalizabile, since the derivative interaction of the $X_{1,2}$-system $\sim\left(X_{1}+X_{2}\right) \square\left(\phi^{\dagger} \phi\right)$ violates power-counting renormalizability as a consequence of the fact that the combination $X \equiv X_{1}+X_{2}$ has a propagator falling down as $1 / p^{2}$ for large $p$ at $z \neq 0$, as can be seen from eq. (C.1):

$$
\Delta_{X X}=\Delta_{X_{1} X_{1}}+\Delta_{X_{2} X_{2}} \sim-\frac{i z}{1+z} \frac{1}{p^{2}}
$$

On the other hand at $z=0 \Delta_{X X}$ goes as $1 / p^{4}$ for large momenta and this compensates the two momenta from the $X \phi^{\dagger} \phi$ interaction vertex, giving rise to a power-counting renormalizable model (at zero $g_{i}$ 's) [20]. 


\section{C.2 The gauge and ghost sector}

The diagonalization in the gauge sector is obtained by redefining the Nakanishi-Lautrup multiplier field

$$
b^{\prime}=b-\frac{1}{\xi} \partial A-e v \chi
$$

Then, the $A_{\mu}$-propagator is

$$
\Delta_{\mu \nu}=-i\left(\frac{1}{p^{2}-M_{A}^{2}} T_{\mu \nu}+\frac{1}{\frac{1}{\xi} p^{2}-M_{A}^{2}}\right) ; \quad M_{A}=e v,
$$

whereas the the Nakanishi-Lautrup, pseudo-Goldstone and ghost propagators are

$$
\Delta_{b^{\prime} b^{\prime}}=i \frac{1}{\xi} ; \quad \Delta_{\chi \chi}=\frac{i}{p^{2}-\xi M_{A}} ; \quad \Delta_{\bar{\omega} \omega}=\frac{i}{p^{2}-\xi M_{A}^{2}}
$$

As usual, $\xi=0$ corresponds to the Landau gauge, whereas $\xi=1$ is the Feynman gauge.

Finally, the ghost associated to the constraint BRST symmetry is free:

$$
\Delta_{\bar{c} c}=\frac{-i}{p^{2}-m^{2}} .
$$

\section{List of gauge-invariant operators}

\section{D.1 Pure external sources invariants}

$$
\begin{aligned}
& \vartheta_{1} \int \mathrm{d}^{4} x \bar{c}^{*} ; \quad \vartheta_{2} \int \mathrm{d}^{4} x T_{1} ; \quad \vartheta_{3} \int \mathrm{d}^{4} x U ; \quad \vartheta_{4} \int \mathrm{d}^{4} x R, \\
& \frac{\vartheta_{5}}{2} \int \mathrm{d}^{4} x\left(\bar{c}^{*}\right)^{2} ; \quad \frac{\vartheta_{6}}{2} \int \mathrm{d}^{4} x T_{1}^{2} ; \quad \frac{\vartheta_{7}}{2} \int \mathrm{d}^{4} x U^{2} ; \quad \frac{\vartheta_{8}}{2} \int \mathrm{d}^{4} x R^{2}, \\
& \frac{\vartheta_{9}}{2} \int \mathrm{d}^{4} x \bar{c}^{*} \square \bar{c}^{*} ; \quad \frac{\vartheta_{10}}{2} \int \mathrm{d}^{4} x T_{1} \square T_{1} ; \quad \frac{\vartheta_{11}}{2} \int \mathrm{d}^{4} x U \square U ; \quad \frac{\vartheta_{12}}{2} \int \mathrm{d}^{4} x R \square R, \\
& \vartheta_{13} \int \mathrm{d}^{4} x \bar{c}^{*} T_{1} ; \quad \vartheta_{14} \int \mathrm{d}^{4} x \bar{c}^{*} U ; \quad \vartheta_{15} \int \mathrm{d}^{4} x \bar{c}^{*} R ; \quad \vartheta_{16} \int \mathrm{d}^{4} x T_{1} U, \\
& \vartheta_{17} \int \mathrm{d}^{4} x T_{1} R ; \quad \vartheta_{18} \int \mathrm{d}^{4} x U R ; \quad \vartheta_{19} \int \mathrm{d}^{4} x \bar{c}^{*} \square T_{1} ; \quad \vartheta_{20} \int \mathrm{d}^{4} x \bar{c}^{*} \square U, \\
& \vartheta_{21} \int \mathrm{d}^{4} x \bar{c}^{*} \square R ; \quad \vartheta_{22} \int \mathrm{d}^{4} x T_{1} \square U ; \quad \vartheta_{23} \int \mathrm{d}^{4} x T_{1} \square R ; \quad \vartheta_{24} \int \mathrm{d}^{4} x U \square R, \\
& \frac{\vartheta_{25}}{6} \int \mathrm{d}^{4} x\left(\bar{c}^{*}\right)^{3} ; \quad \frac{\vartheta_{26}}{6} \int \mathrm{d}^{4} x T_{1}^{3} ; \quad \frac{\vartheta_{27}}{6} \int \mathrm{d}^{4} x U^{3} ; \quad \frac{\vartheta_{28}}{6} \int \mathrm{d}^{4} x R^{3}, \\
& \frac{\vartheta_{29}}{2} \int \mathrm{d}^{4} x\left(\bar{c}^{*}\right)^{2} T_{1} ; \quad \frac{\vartheta_{30}}{2} \int \mathrm{d}^{4} x\left(\bar{c}^{*}\right)^{2} U ; \quad \frac{\vartheta_{31}}{2} \int \mathrm{d}^{4} x\left(\bar{c}^{*}\right)^{2} R ; \quad \frac{\vartheta_{32}}{2} \int \mathrm{d}^{4} x \bar{c}^{*} T_{1}^{2}, \\
& \frac{\vartheta_{33}}{2} \int \mathrm{d}^{4} x \bar{c}^{*} U^{2} ; \quad \frac{\vartheta_{34}}{2} \int \mathrm{d}^{4} x \bar{c}^{*} R^{2} ; \quad \vartheta_{35} \int \mathrm{d}^{4} x \bar{c}^{*} T_{1} U ; \quad \vartheta_{36} \int \mathrm{d}^{4} x \bar{c}^{*} T_{1} R, \\
& \vartheta_{37} \int \mathrm{d}^{4} x \bar{c}^{*} U R ; \quad \frac{\vartheta_{38}}{2} \int \mathrm{d}^{4} x T_{1}^{2} U ; \quad \frac{\vartheta_{39}}{2} \int \mathrm{d}^{4} x T_{1}^{2} R ; \quad \frac{\vartheta_{40}}{2} \int \mathrm{d}^{4} x T_{1} U^{2}, \\
& \frac{\vartheta_{41}}{2} \int \mathrm{d}^{4} x T_{1} R^{2} ; \quad \vartheta_{42} \int \mathrm{d}^{4} x T_{1} U R ; \quad \frac{\vartheta_{43}}{2} \int \mathrm{d}^{4} x U^{2} R ; \quad \frac{\vartheta_{44}}{2} \int \mathrm{d}^{4} x U R^{2} .
\end{aligned}
$$




\section{D.2 Mixed field-external sources invariants}
$\theta_{1} \int \mathrm{d}^{4} x \bar{c}^{*}\left(\phi^{\dagger} \phi-\frac{v^{2}}{2}\right) ;$
$\theta_{2} \int \mathrm{d}^{4} x T_{1}\left(\phi^{\dagger} \phi-\frac{v^{2}}{2}\right) ;$
$\theta_{3} \int \mathrm{d}^{4} x U\left(\phi^{\dagger} \phi-\frac{v^{2}}{2}\right)$,
$\theta_{4} \int \mathrm{d}^{4} x R\left(\phi^{\dagger} \phi-\frac{v^{2}}{2}\right)$
$\theta_{5} \int \mathrm{d}^{4} x \bar{c}^{*}\left(D^{\mu} \phi\right)^{\dagger} D_{\mu} \phi ;$
$\theta_{6} \int \mathrm{d}^{4} x T_{1}\left(D^{\mu} \phi\right)^{\dagger} D_{\mu} \phi$,
$\theta_{7} \int \mathrm{d}^{4} x U\left(D^{\mu} \phi\right)^{\dagger} D_{\mu} \phi$
$\theta_{8} \int \mathrm{d}^{4} x R\left(D^{\mu} \phi\right)^{\dagger} D_{\mu} \phi$
$\theta_{9} \int \mathrm{d}^{4} x \bar{c}^{*}\left(\phi^{\dagger} D^{2} \phi+\right.$ h.c. $)$,

$\theta_{10} \int \mathrm{d}^{4} x T_{1}\left(\phi^{\dagger} D^{2} \phi+\right.$ h.c. $) ; \quad \theta_{11} \int \mathrm{d}^{4} x U\left(\phi^{\dagger} D^{2} \phi+\right.$ h.c. $) ; \quad \theta_{12} \int \mathrm{d}^{4} x R\left(\phi^{\dagger} D^{2} \phi+\right.$ h.c. $)$,

$\frac{\theta_{13}}{2} \int \mathrm{d}^{4} x \bar{c}^{*}\left(\phi^{\dagger} \phi-\frac{v^{2}}{2}\right)^{2} ; \quad \frac{\theta_{14}}{2} \int \mathrm{d}^{4} x T_{1}\left(\phi^{\dagger} \phi-\frac{v^{2}}{2}\right)^{2} ; \quad \frac{\theta_{15}}{2} \int \mathrm{d}^{4} x U\left(\phi^{\dagger} \phi-\frac{v^{2}}{2}\right)^{2}$,

$\frac{\theta_{16}}{2} \int \mathrm{d}^{4} x R\left(\phi^{\dagger} \phi-\frac{v^{2}}{2}\right)^{2} ; \quad \theta_{17} \int \mathrm{d}^{4} x \bar{c}^{*} F_{\mu \nu}^{2} ; \quad \theta_{18} \int \mathrm{d}^{4} x T_{1} F_{\mu \nu}^{2}$,

$\theta_{19} \int \mathrm{d}^{4} x U F_{\mu \nu}^{2}$

$\theta_{20} \int \mathrm{d}^{4} x R F_{\mu \nu}^{2}$

$\frac{\theta_{21}}{2} \int \mathrm{d}^{4} x\left(\bar{c}^{*}\right)^{2}\left(\phi^{\dagger} \phi-\frac{v^{2}}{2}\right)$,

$\frac{\theta_{22}}{2} \int \mathrm{d}^{4} x T_{1}^{2}\left(\phi^{\dagger} \phi-\frac{v^{2}}{2}\right) ; \quad \frac{\theta_{23}}{2} \int \mathrm{d}^{4} x U^{2}\left(\phi^{\dagger} \phi-\frac{v^{2}}{2}\right) ; \quad \frac{\theta_{24}}{2} \int \mathrm{d}^{4} x R^{2}\left(\phi^{\dagger} \phi-\frac{v^{2}}{2}\right)$,

$\theta_{25} \int \mathrm{d}^{4} x \bar{c}^{*} T_{1}\left(\phi^{\dagger} \phi-\frac{v^{2}}{2}\right) ; \quad \theta_{26} \int \mathrm{d}^{4} x \bar{c}^{*} U\left(\phi^{\dagger} \phi-\frac{v^{2}}{2}\right) ; \quad \theta_{27} \int \mathrm{d}^{4} x \bar{c}^{*} R\left(\phi^{\dagger} \phi-\frac{v^{2}}{2}\right)$,

$\theta_{28} \int \mathrm{d}^{4} x T_{1} U\left(\phi^{\dagger} \phi-\frac{v^{2}}{2}\right) ; \quad \theta_{29} \int \mathrm{d}^{4} x T_{1} R\left(\phi^{\dagger} \phi-\frac{v^{2}}{2}\right) ; \quad \theta_{30} \int \mathrm{d}^{4} x U R\left(\phi^{\dagger} \phi-\frac{v^{2}}{2}\right)$.

\section{D.3 Gauge invariants depending only on the fields}

$$
\begin{array}{ll}
\lambda_{1} \int \mathrm{d}^{4} x\left(\phi^{\dagger} \phi-\frac{v^{2}}{2}\right) ; & \lambda_{2} \int \mathrm{d}^{4} x\left(\phi^{\dagger} \phi-\frac{v^{2}}{2}\right)^{2}, \\
\lambda_{3} \int \mathrm{d}^{4} x\left(\phi^{\dagger} \phi-\frac{v^{2}}{2}\right)^{3} ; & \lambda_{4} \int \mathrm{d}^{4} x\left(D^{\mu} \phi\right)^{\dagger} D_{\mu} \phi, \\
\lambda_{5} \int \mathrm{d}^{4} x\left(\phi^{\dagger} D^{(\mu \nu \mu \nu)} \phi+\text { h.c. }\right) ; & \lambda_{6} \int \mathrm{d}^{4} x\left(\phi^{\dagger} \phi-\frac{v^{2}}{2}\right)\left(\phi^{\dagger} D^{2} \phi+\text { h.c. }\right), \\
\lambda_{7} \int \mathrm{d}^{4} x\left(\phi^{\dagger} \phi-\frac{v^{2}}{2}\right)\left(D^{\mu} \phi\right)^{\dagger} D_{\mu} \phi ; & \lambda_{8} \int \mathrm{d}^{4} x F_{\mu \nu}^{2}, \\
\lambda_{9} \int \mathrm{d}^{4} x \partial^{\rho} F_{\rho \mu} \partial_{\sigma} F^{\sigma \mu} ; & \lambda_{10} \int \mathrm{d}^{4} x F_{\mu \nu}^{2}\left(\phi^{\dagger} \phi-\frac{v^{2}}{2}\right),
\end{array}
$$


where $D^{(\mu \nu \mu \nu)}$ denotes complete symmetrization over $\mu, \nu$ :

$$
D^{(\mu \nu \mu \nu)} \phi \equiv\left[\left(D^{2}\right)^{2}+D^{\mu} D^{\nu} D_{\mu} D_{\nu}+D^{\mu} D^{2} D_{\mu}\right] \phi .
$$

Notice that in the text we have denoted by $\mathscr{I}_{j}$ the invariant with coefficient $\lambda_{j}$.

\section{E On-shell reduction of dim. 6 field-dependent gauge invariant operators}

We consider in this appendix the on-shell reduction of dimension 6 operators in the target theory. The relevant classical gauge-invariant action $S$ is obtained from the first four lines of eq. (2.1) by going on-shell with $X_{1,2}$.

The corresponding equations of motion for the gauge field and the scalar $\phi$ are

$$
\begin{aligned}
\frac{\delta S}{\delta A_{\mu}} & =\partial^{\rho} F_{\rho \mu}+i\left[\phi^{\dagger} D_{\mu} \phi-\left(D_{\mu} \phi\right)^{\dagger} \phi\right], \\
\frac{\delta S}{\delta \phi} & =-\left(D^{2} \phi\right)^{\dagger}-\frac{M^{2}}{v^{2}}\left(\phi^{\dagger} \phi-\frac{v^{2}}{2}\right) \phi^{\dagger}, \\
\frac{\delta S}{\delta \phi^{\dagger}} & =-\left(D^{2} \phi\right)-\frac{M^{2}}{v^{2}}\left(\phi^{\dagger} \phi-\frac{v^{2}}{2}\right) \phi .
\end{aligned}
$$

Since we will be interested only in the one-loop corrections that are linear in the $g_{i}$ 's and $z$ we can limit ourselves to the leading order equations of motion in eq. (E.1); also we recall here the identity

$$
\left[D_{\mu}, D_{\nu}\right]=-i F_{\mu \nu}
$$

The on-shell independent dimension 6 operators can be chosen to be $\mathscr{I}_{3}, \mathscr{I}_{7}$ and $\mathscr{I}_{10}$. Notice that the operator in the tree-level vertex functional

$$
\begin{aligned}
\int \mathrm{d}^{4} x\left(\phi^{\dagger} \phi-\frac{v^{2}}{2}\right) & \square\left(\phi^{\dagger} \phi-\frac{v^{2}}{2}\right) \\
& =\int \mathrm{d}^{4} x\left(\phi^{\dagger} \phi-\frac{v^{2}}{2}\right)\left[\left(D^{2} \phi\right)^{\dagger} \phi+\phi^{\dagger}\left(D^{2} \phi\right)+2\left(D^{\mu} \phi\right)^{\dagger} D_{\mu} \phi\right],
\end{aligned}
$$

can be represented in terms of invariants in the contractible pairs basis as in the r.h.s. of the above equation. Therefore we just need to reduce all $\mathscr{I}_{i}$ 's invariants in terms of $\mathscr{I}_{3}, \mathscr{I}_{7}$ and $\mathscr{I}_{10}$ by using the equations of motion (E.1).

Let us start from $\mathscr{I}_{5}$. This operator contains three terms, namely:

$$
\int \mathrm{d}^{4} x \phi^{\dagger} D^{4} \phi ; \quad \int \mathrm{d}^{4} x \phi^{\dagger} D^{\mu} D^{2} D_{\mu} \phi ; \quad \int \mathrm{d}^{4} x \phi^{\dagger} D^{\mu} D^{\nu} D_{\mu} D_{\nu} \phi .
$$

Then one finds that:

- Integration by parts gives:

$$
\int \mathrm{d}^{4} x \phi^{\dagger}\left(D^{2}\right)^{2} \phi=\int \mathrm{d}^{4} x\left(D^{2} \phi\right)^{\dagger} D^{2} \phi \sim \int \mathrm{d}^{4} x \frac{M^{4}}{v^{4}}\left(\phi^{\dagger} \phi-\frac{v^{2}}{2}\right)^{2} \phi^{\dagger} \phi
$$


where the equations of motion for $\phi, \phi^{\dagger}$ have been used in the last line. Hence we obtain

$$
\begin{aligned}
\int \mathrm{d}^{4} x \phi^{\dagger}\left(D^{2}\right)^{2} \phi & \sim \int \mathrm{d}^{4} x\left\{\frac{M^{4}}{v^{4}}\left(\phi^{\dagger} \phi-\frac{v^{2}}{2}\right)^{3}+\frac{M^{4}}{2 v^{2}}\left(\phi^{\dagger} \phi-\frac{v^{2}}{2}\right)^{2}\right\} \\
& =\frac{M^{4}}{2 v^{2}} \mathscr{I}_{2}+\frac{M^{4}}{v^{4}} \mathscr{I}_{3} .
\end{aligned}
$$

- The second term can be rewritten as follows

$$
\begin{aligned}
\int \mathrm{d}^{4} x \phi^{\dagger} D^{\mu} D^{2} D_{\mu} \phi & =\int \mathrm{d}^{4} x \phi^{\dagger} D^{\mu} D^{\rho} D_{\mu} D_{\rho} \phi+\int \mathrm{d}^{4} x \phi^{\dagger} D^{\mu} D^{\rho}\left[D_{\rho}, D_{\mu}\right] \phi \\
& =\int \mathrm{d}^{4} x \phi^{\dagger} D^{\mu} D^{\rho} D_{\mu} D_{\rho} \phi-i \int \mathrm{d}^{4} x\left(D^{\rho} D^{\mu} \phi\right)^{\dagger} F_{\rho \mu} \phi,
\end{aligned}
$$

where in the last line we have used eq. (E.2) and integrated by parts. Now

$$
-i \int \mathrm{d}^{4} x\left(D^{\rho} D^{\mu} \phi\right)^{\dagger} F_{\rho \mu} \phi=-\frac{i}{2} \int \mathrm{d}^{4} x\left(\left[D^{\rho}, D^{\mu}\right] \phi\right)^{\dagger} F_{\rho \mu} \phi=-\frac{1}{2} \int \mathrm{d}^{4} x F_{\rho \mu}^{2} \phi^{\dagger} \phi,
$$

again by using eq. (E.2). Eventually we arrive at the result

$$
\begin{aligned}
\int \mathrm{d}^{4} x \phi^{\dagger} D^{\mu} D^{2} D_{\mu} \phi= & \int \mathrm{d}^{4} x \phi^{\dagger} D^{\mu} D^{\rho} D_{\mu} D_{\rho} \phi \\
& -\frac{1}{2} \int \mathrm{d}^{4} x F_{\rho \mu}^{2}\left(\phi^{\dagger} \phi-\frac{v^{2}}{2}\right)-\frac{v^{2}}{4} \int \mathrm{d}^{4} x F_{\rho \mu}^{2} \\
= & \int \mathrm{d}^{4} x \phi^{\dagger} D^{\mu} D^{\rho} D_{\mu} D_{\rho} \phi-\frac{v^{2}}{4} \mathscr{I}_{8}-\frac{1}{2} \mathscr{J}_{10} .
\end{aligned}
$$

- We are finally left with the decomposition of the last term in eq. (E.4). One has

$$
\begin{aligned}
\int \mathrm{d}^{4} x \phi^{\dagger} D^{\mu} D^{\rho} D_{\mu} D_{\rho} \phi & =\int \mathrm{d}^{4} x\left[\phi^{\dagger} D^{4} \phi+\phi^{\dagger} D^{\mu}\left[D^{\rho}, D_{\mu}\right] D_{\rho} \phi\right] \\
& =\int \mathrm{d}^{4} x\left[\left(D^{2} \phi\right)^{\dagger} D^{2} \phi-i F_{\mu \rho}\left(D^{\mu} \phi\right)^{\dagger} D^{\rho} \phi\right],
\end{aligned}
$$

where we have used eq. (E.2) and integrated by parts. It is convenient to split the last term in the above equation as follows

$$
\begin{aligned}
i \int \mathrm{d}^{4} x F_{\mu \rho}\left(D^{\mu} \phi\right)^{\dagger} D^{\rho} \phi & =\frac{i}{2} \int \mathrm{d}^{4} x F_{\mu \rho}\left\{\left(D^{\mu} \phi\right)^{\dagger} D^{\rho} \phi+\left(D^{\mu} \phi\right)^{\dagger} D^{\rho} \phi\right\} \\
& =\int \mathrm{d}^{4} x\left\{-\frac{i}{2} \partial^{\rho} F_{\rho \mu}\left[\phi^{\dagger} D^{\mu} \phi-\left(D^{\mu} \phi\right)^{\dagger} \phi\right]\right. \\
& \left.\quad-\frac{i}{4} F_{\mu \rho}\left[\phi^{\dagger}\left[D^{\mu}, D^{\rho}\right] \phi+\left(\left[D^{\rho}, D^{\mu}\right] \phi\right)^{\dagger} \phi\right]\right\} \\
& =\int \mathrm{d}^{4} x\left\{-\frac{i}{2} \partial^{\rho} F_{\rho \mu}\left[\phi^{\dagger} D^{\mu} \phi-\left(D^{\mu} \phi\right)^{\dagger} \phi\right]-\frac{1}{2} F_{\mu \rho}^{2} \phi^{\dagger} \phi\right\} .
\end{aligned}
$$


By using the $A_{\mu}$-equation of motion (E.1) the first term in the last line of the above equation becomes

$$
\begin{aligned}
-\frac{i}{2} \int \mathrm{d}^{4} x & \partial^{\rho} F_{\rho \mu}\left[\phi^{\dagger} D^{\mu} \phi-\left(D^{\mu} \phi\right)^{\dagger} \phi\right] \\
& \sim-\frac{1}{2} \int \mathrm{d}^{4} x\left(\phi^{\dagger} D^{\mu} \phi-\left(D^{\mu} \phi\right)^{\dagger} \phi\right)\left(\phi^{\dagger} D_{\mu} \phi-\left(D_{\mu} \phi\right)^{\dagger} \phi\right) \\
& =\int \mathrm{d}^{4} x\left\{\phi^{\dagger} \phi\left(D^{\mu} \phi\right)^{\dagger} D_{\mu} \phi-\frac{1}{2}\left[\phi^{\dagger} D_{\mu} \phi \phi^{\dagger} D^{\mu} \phi+\text { h.c. }\right]\right\} .
\end{aligned}
$$

Integrating by parts the last term in the last line of the above equation one finds

$$
\begin{aligned}
&-\frac{1}{2} \int \mathrm{d}^{4} x {\left[\phi^{\dagger} D_{\mu} \phi \phi^{\dagger} D^{\mu} \phi+\text { h.c. }\right] } \\
&=\int \mathrm{d}^{4} x\left\{2 \phi^{\dagger} \phi\left(D^{\mu} \phi\right)^{\dagger} D_{\mu} \phi+\frac{1}{2} \phi^{\dagger} \phi\left[\phi^{\dagger} D^{2} \phi+\left(D^{2} \phi\right)^{\dagger} \phi\right]\right\},
\end{aligned}
$$

and thus

$$
\begin{aligned}
-\frac{i}{2} \int \mathrm{d}^{4} x & \partial^{\rho} F_{\rho \mu}\left[\phi^{\dagger} D^{\mu} \phi-\left(D^{\mu} \phi\right)^{\dagger} \phi\right] \\
& \sim-\frac{1}{2} \int \mathrm{d}^{4} x\left(\phi^{\dagger} D^{\mu} \phi-\left(D^{\mu} \phi\right)^{\dagger} \phi\right)\left(\phi^{\dagger} D_{\mu} \phi-\left(D_{\mu} \phi\right)^{\dagger} \phi\right) \\
& =\int \mathrm{d}^{4} x\left\{3 \phi^{\dagger} \phi\left(D^{\mu} \phi\right)^{\dagger} D_{\mu} \phi+\frac{1}{2} \phi^{\dagger} \phi\left[\phi^{\dagger} D^{2} \phi+\left(D^{2} \phi\right)^{\dagger} \phi\right]\right\} .
\end{aligned}
$$

Putting everything together we find

$$
\begin{aligned}
& \int \mathrm{d}^{4} x \phi^{\dagger} D^{\mu} D^{\rho} D_{\mu} D_{\rho} \phi \\
&=\int \mathrm{d}^{4} x\left\{\left(D^{2} \phi\right)^{\dagger} D^{2} \phi-3 \phi^{\dagger} \phi\left(D^{\mu} \phi\right)^{\dagger} D_{\mu} \phi\right. \\
&\left.\quad-\frac{1}{2} \phi^{\dagger} \phi\left[\phi^{\dagger} D^{2} \phi+\left(D^{2} \phi\right)^{\dagger} \phi\right]+\frac{1}{2} F_{\mu \rho}^{2} \phi^{\dagger} \phi\right\} \\
& \sim \int \mathrm{d}^{4} x\left\{\frac{M^{2}}{v^{2}}\left(1+\frac{M^{2}}{v^{2}}\right)\left(\phi^{\dagger} \phi-\frac{v^{2}}{2}\right)^{3}-3\left(\phi^{\dagger} \phi-\frac{v^{2}}{2}\right)\left(D^{\mu} \phi\right)^{\dagger} D_{\mu} \phi\right. \\
& \quad+\frac{1}{2} F_{\mu \rho}^{2}\left(\phi^{\dagger} \phi-\frac{v^{2}}{2}\right)-\frac{3 v^{2}}{2}\left(D^{\mu} \phi\right)^{\dagger} D_{\mu} \phi+M^{2}\left(1+\frac{M^{2}}{2 v^{2}}\right)\left(\phi^{\dagger} \phi-\frac{v^{2}}{2}\right)^{2} \\
&\left.\quad+\frac{1}{4} M^{2} v^{2}\left(\phi^{\dagger} \phi-\frac{v^{2}}{2}\right)+\frac{v^{2}}{4} F_{\mu \rho}^{2}\right\} \\
&=\frac{1}{4} M^{2} v^{2} \mathscr{I}_{1}+M^{2}\left(1+\frac{M^{2}}{2 v^{2}}\right) \mathscr{I}_{2}+\frac{M^{2}}{v^{2}}\left(1+\frac{M^{2}}{v^{2}}\right) \mathscr{I}_{3}-\frac{3}{2} v^{2} \mathscr{I}_{4} \\
& \quad-3 \mathscr{I}_{7}+\frac{v^{2}}{4} \mathscr{I}_{8}+\frac{1}{2} \mathscr{J}_{10} .
\end{aligned}
$$

By using eqs. (E.6), (E.8) and (E.14) we obtain

$$
\begin{aligned}
\mathscr{I}_{5} \sim & \frac{1}{2} M^{2} v^{2} \mathscr{I}_{1}+M^{2}\left(2+\frac{3}{2} \frac{M^{2}}{v^{2}}\right) \mathscr{I}_{2}+\frac{M^{2}}{v^{2}}\left(2+\frac{3 M^{2}}{v^{2}}\right) \mathscr{I}_{3} \\
& -3 v^{2} \mathscr{I}_{4}-6 \mathscr{I}_{7}+\frac{1}{4} v^{2} \mathscr{I}_{8}+\frac{1}{2} \mathscr{I}_{10} .
\end{aligned}
$$


We now move to $\mathscr{I}_{6}$. By using the equations of motion for $\phi, \phi^{\dagger}$ in eq. (E.1) we find

$$
\mathscr{I}_{6} \sim \int \mathrm{d}^{4} x\left\{-2 \frac{M^{2}}{v^{2}}\left(\phi^{\dagger} \phi-\frac{v^{2}}{2}\right)^{3}-M^{2}\left(\phi^{\dagger} \phi-\frac{v^{2}}{2}\right)^{2}\right\}=-2 \frac{M^{2}}{v^{2}} \mathscr{I}_{3}-M^{2} \mathscr{I}_{2}
$$

Finally we need to consider $\mathscr{F}_{9}$. Use of $A_{\mu}$-equation of motion yields

$$
\begin{aligned}
\mathscr{I}_{9} & \sim-\int \mathrm{d}^{4} x\left[\phi^{\dagger} D_{\mu} \phi-\left(D_{\mu} \phi\right)^{\dagger} \phi\right]^{2} \\
& \sim \int \mathrm{d}^{4} x \phi^{\dagger} \phi\left[6\left(D^{\mu} \phi\right)^{\dagger} D_{\mu} \phi+\phi^{\dagger} D^{2} \phi+\left(D^{2} \phi\right)^{\dagger} \phi\right] \\
& =\int \mathrm{d}^{4} x\left[\left(\phi^{\dagger} \phi-\frac{v^{2}}{2}\right)+\frac{v^{2}}{2}\right]\left\{6\left(D^{\mu} \phi\right)^{\dagger} D_{\mu} \phi-2 \frac{M^{2}}{v^{2}}\left(\phi^{\dagger} \phi-\frac{v^{2}}{2}\right)^{2}-M^{2}\left(\phi^{\dagger} \phi-\frac{v^{2}}{2}\right)\right\} \\
& =-\frac{M^{2} v^{2}}{2} \mathscr{J}_{1}-2 M^{2} \mathscr{I}_{2}-2 \frac{M^{2}}{v^{2}} \mathscr{J}_{3}+3 v^{2} \mathscr{I}_{4}+6 \mathscr{I}_{7} .
\end{aligned}
$$

Open Access. This article is distributed under the terms of the Creative Commons Attribution License (CC-BY 4.0), which permits any use, distribution and reproduction in any medium, provided the original author(s) and source are credited.

\section{References}

[1] J. Gomis and S. Weinberg, Are nonrenormalizable gauge theories renormalizable?, Nucl. Phys. B 469 (1996) 473 [hep-th/9510087] [InSPIRE].

[2] J.C. Collins and R.J. Scalise, The renormalization of composite operators in Yang-Mills theories using general covariant gauge, Phys. Rev. D 50 (1994) 4117 [hep-ph/9403231] [INSPIRE].

[3] S.D. Joglekar and B.W. Lee, General theory of renormalization of gauge invariant operators, Annals Phys. 97 (1976) 160 [INSPIRE].

[4] H. Kluberg-Stern and J.B. Zuber, Ward identities and some clues to the renormalization of gauge invariant operators, Phys. Rev. D 12 (1975) 467 [INSPIRE].

[5] H. Kluberg-Stern and J.B. Zuber, Renormalization of nonabelian gauge theories in a background field gauge. 1. Green functions, Phys. Rev. D 12 (1975) 482 [INSPIRE].

[6] H. Kluberg-Stern and J.B. Zuber, Renormalization of nonabelian gauge theories in a background field gauge. 2. Gauge invariant operators, Phys. Rev. D 12 (1975) 3159 [INSPIRE].

[7] G. Barnich, F. Brandt and M. Henneaux, Local BRST cohomology in gauge theories, Phys. Rept. 338 (2000) 439 [hep-th/0002245] [INSPIRE].

[8] E.E. Jenkins, A.V. Manohar and M. Trott, Renormalization group evolution of the standard model dimension six operators. Parti I. Formalism and $\lambda$ dependence, JHEP 10 (2013) 087 [arXiv: 1308.2627] [INSPIRE].

[9] E.E. Jenkins, A.V. Manohar and M. Trott, Renormalization group evolution of the standard model dimension six operators. Part II. Yukawa dependence, JHEP 01 (2014) 035 [arXiv: 1310.4838$]$ [INSPIRE].

[10] LHC Higgs Cross Section Working Group collaboration, Handbook of LHC Higgs Cross Sections: 4. Deciphering the nature of the Higgs sector, arXiv:1610.07922 [INSPIRE]. 
[11] I. Brivio and M. Trott, The standard model as an effective field theory, Phys. Rept. 793 (2019) 1 [arXiv: 1706.08945] [INSPIRE].

[12] B. Grzadkowski, M. Iskrzynski, M. Misiak and J. Rosiek, Dimension-six terms in the standard model Lagrangian, JHEP 10 (2010) 085 [arXiv: 1008.4884] [INSPIRE].

[13] M.E. Machacek and M.T. Vaughn, Two loop renormalization group equations in a general quantum field theory. 1. Wave function renormalization, Nucl. Phys. B 222 (1983) 83 [INSPIRE].

[14] M.E. Machacek and M.T. Vaughn, Two loop renormalization group equations in a general quantum field theory. 2. Yukawa couplings, Nucl. Phys. B 236 (1984) 221 [INSPIRE].

[15] R. Grzywacz et al., Isomeric states in 66As, Phys. Lett. B 429 (1998) 247 [InSPIRE].

[16] D. Binosi and A. Quadri, Off-shell renormalization in the presence of dimension 6 derivative operators. Part I. General theory, JHEP 09 (2019) 032 [arXiv: 1904.06692] [INSPIRE].

[17] D. Binosi and A. Quadri, Off-shell renormalization in the presence of dimension 6 derivative operators. Part II. UV coefficients, arXiv:1904.06693 [INSPIRE].

[18] D. Binosi and A. Quadri, in preparation.

[19] A. Quadri, The Abelian embedding formulation of the Stuckelberg model and its power-counting renormalizable extension, Phys. Rev. D 73 (2006) 065024 [hep-th/0601169] [INSPIRE].

[20] A. Quadri, Higgs potential from derivative interactions, Int. J. Mod. Phys. A 32 (2017) 1750089 [arXiv: 1610.00150] [INSPIRE].

[21] T. Hahn, Generating Feynman diagrams and amplitudes with FeynArts 3, Comput. Phys. Commun. 140 (2001) 418 [hep-ph/0012260] [INSPIRE].

[22] T. Hahn, Automatic loop calculations with FeynArts, FormCalc and LoopTools, Nucl. Phys. Proc. Suppl. 89 (2000) 231 [hep-ph/0005029] [InSPIRE].

[23] M. Sperling, D. Stöckinger and A. Voigt, Renormalization of vacuum expectation values in spontaneously broken gauge theories, JHEP 07 (2013) 132 [arXiv:1305.1548] [INSPIRE].

[24] J. Elias-Miró, J.R. Espinosa, E. Masso and A. Pomarol, Renormalization of dimension-six operators relevant for the Higgs decays $h \rightarrow \gamma \gamma, \gamma Z$, JHEP 08 (2013) 033 [arXiv:1302.5661] [INSPIRE].

[25] C. Cheung and C.-H. Shen, Nonrenormalization theorems without supersymmetry, Phys. Rev. Lett. 115 (2015) 071601 [arXiv: 1505.01844] [INSPIRE]. 